\title{
Nuclear morphology is a deep learning biomarker of senescence across tissues and species
}

\author{
Indra Heckenbach
}

University of Copenhagen

\section{Garik Mkrtchyan}

University of Copenhagen

\section{Michael Ben Ezra}

University of Copenhagen

\section{Daniela Bakula}

University of Copenhagen

Jakob Madsen

University of Copenhagen https://orcid.org/0000-0002-2841-7284

Malte Nielsen

Gubra

Denise Oró

Gubra

M Laura Idda

National Institute on Aging

Myriam Gorospe

Nat Inst on Aging, NIH

\section{Laust Mortensen}

University of Copenhagen

\section{Eric Verdin}

Buck Institute for Research on Aging

\section{Rudi Westendorp}

University of Copenhagen

Morten Scheibye-Knudsen ( $\nabla$ mscheibye@sund.ku.dk)

University of Copenhagen https://orcid.org/0000-0002-6637-1280

\section{Article}

Keywords: cellular senescence, nuclear morphology, age-related diseases

Posted Date: November 11th, 2021 
DOI: https://doi.org/10.21203/rs.3.rs-1017512/v1

License: (c) (1) This work is licensed under a Creative Commons Attribution 4.0 International License. Read Full License

Version of Record: A version of this preprint was published at Nature Aging on August 15th, 2022. See the published version at https://doi.org/10.1038/s43587-022-00263-3. 


\section{Nuclear morphology is a deep learning biomarker of senescence \\ 2 across tissues and species}

5 Indra Heckenbach ${ }^{1,2,3}$, Garik V Mkrtchyan ${ }^{1}$, Michael Ben Ezra ${ }^{1,4}$, Daniela Bakula ${ }^{1}$, Jakob Sture

6 Madsen $^{1}$, Malte Hasle Nielsen ${ }^{5}$, Denise Oró $^{5}$, M Laura Idda $^{6,7}$, Myriam Gorospe ${ }^{6}$, Laust

7 Mortensen ${ }^{4,8}$, Eric Verdin ${ }^{2}$, Rudi Westendorp ${ }^{4,8}$, Morten Scheibye-Knudsen ${ }^{1,3^{*}}$

8

$9{ }^{1}$ Center for Healthy Aging, Department of Cellular and Molecular Medicine, University of Copenhagen,

10 Copenhagen, Denmark

$11{ }^{2}$ Buck Institute for Research on Aging, Novato, CA, USA

$12{ }^{3}$ Tracked.bio, Copenhagen, Denmark

$13 \quad{ }^{4}$ Methods and Analysis, Statistics Denmark, Copenhagen, Denmark

$14{ }^{5}$ Gubra, Hørsholm, Denmark

$15{ }^{6}$ Laboratory of Genetics and Genomics, National Institute on Aging Intramural Research Program,

16 National Institutes of Health, Baltimore, MD, USA

$17{ }^{7}$ Istituto di Ricerca Genetica e Biomedica, Consiglio Nazionale delle Ricerche, Sassari, Italy

$18{ }^{8}$ Department of Public Health, University of Copenhagen, Copenhagen, Denmark

19 *Correspondence: mscheibye@sund.ku.dk

20

21

22 


\section{Abstract}

25 Cellular senescence is a critical component of aging and many age-related diseases, but 26 understanding its role in human health is challenging in part due to the lack of exclusive or

27 universal markers. Using neural networks, we achieve high accuracy in predicting senescence

28 state and type from the nuclear morphology of DAPI-stained human fibroblasts, murine

29 astrocytes, murine neurons, and fibroblasts derived from premature aging diseases in culture.

30 After generalizing this approach, the predictor recognizes an increasing rate of senescent cells

31 with age in H\&E-stained murine liver tissue and human dermal biopsies, suggesting that

32 alterations in nuclear morphology is a universal feature of senescence. Evaluating

33 corresponding medical records reveals that individuals with a higher rate of senescent cells

34 have a significantly decreased rate of malignant neoplasms, lending support for the protective

35 role of senescence in limiting cancer development. Additionally, we find a positive association

36 with lower significance for other conditions, including osteoporosis, osteoarthritis, hypertension,

37 cerebral infarction, hyperlipidemia, and hypercholesteremia. In sum, we introduce a predictor of 38 cellular senescence based on nuclear morphology that is applicable across tissues and species 39 and is associated with health outcomes in humans.

40 


\section{Introduction}

44 Cellular senescence is widely recognized as a fundamental process in aging, both as a primary

45 causal factor in the decline of tissue homeostasis and as a consequence of other aging

46 processes such as inflammation and DNA damage ${ }^{1-3}$. Due to its critical role in disease etiology,

47 senescence is increasingly recognized as a target for pharmaceutical intervention ${ }^{4}$. It also

48 serves as a biomarker for aging ${ }^{5}$, possibly providing a more nuanced measure of age-related

49 health in model organisms beyond simple chronological age. However, the role of senescence

50 in human health is not clearly understood. Senescent cells present a complex and diverse

51 phenotype, which varies significantly by cell type and source ${ }^{6,7}$. There is considerable overlap

52 between molecular factors that associate with senescence, DNA damage, inflammation, and

53 other processes ${ }^{8,9}$. Some of the most common markers of senescence are senescence-

54 associated $\beta$-galactosidase activity (SA- $\beta$-gal) and increased levels of the cell cycle inhibitors

$55 \mathrm{p} 16$ and p21. Nevertheless, there is no single marker that reliably and consistently identifies

56 senescence ${ }^{10-12}$. Importantly, senescent cells often exhibit an altered morphology, including

57 expanded nuclei and an irregular, flattened appearance ${ }^{13,14}$, making senescence amenable to

58 analysis with computer vision and machine learning methods ${ }^{15}$.

60 We present deep learning models that can predict cellular senescence with high accuracy

61 based on nuclear morphology. These methods can further distinguish between multiple types of

62 senescence, including radiation-induced damage and replicative exhaustion. Notably, predicted

63 senescence correlates substantially with SA- $\beta$-gal, p16, p21, p53, and DNA damage markers

$64 \gamma \mathrm{H} 2 \mathrm{AX}$ and 53BP1 foci counts. Our senescence predictor was developed using normal human

65 fibroblast lines, but it also identifies increased senescence when applied to multiple types of

66 premature aging diseases, including Hutchinson-Gilford progeria syndrome, ataxia

Page 3 of 44 
67 telangiectasia, and Cockayne syndrome. We also evaluated the predictor on mouse astrocytes

68 and neurons and found it indicates increased senescence in cells subjected to ionizing

69 radiation, confirming its relevance to different cell types and organisms. These methods were

70 further applied to H\&E-stained mouse liver tissue, where we found an increasing rate of

71 senescence with age. In addition, H\&E-stained human tissue sections showed an age-

72 dependent increase in senescence. Using the Danish National Patient Register, which records

73 all ambulatory and in-patient contacts with Danish hospitals, we investigated how predicted

74 senescence relates to human disease. In our study of 169 individuals, we found a highly

75 statistically significant relationship between malignant neoplasm incidence and fewer predicted

76 senescent cells, which fits the hypothesis that senescence is a mechanism to limit cancer ${ }^{16-18}$.

77 While oncogenic events are associated with the formation of senescent cells ${ }^{17}$, we speculate

78 that individuals with higher propensity toward developing senescent cells have reduced

79 formation of malignant neoplasm and are at lower risk of cancer. We also found a weaker

80 association between predicted senescence and other conditions, including osteoporosis,

81 osteoarthritis, hypertension, cerebral infarction, hyperlipidemia, and hypercholesteremia.

\section{Results}

85 Three dermal fibroblast cell lines were induced to senescence by ionizing radiation (IR) or 86 passaged until they reached replicative senescence (RS) (Fig. S1a, b, c). To confirm that the IR

87 treated cells were senescent we evaluated levels of senescence markers p16, p21, p53 and

88 interleukin- 6 by immunohistochemistry and/or qPCR and found that IR led to a significant

89 increase in these markers (Fig. S1d, e, f, g, h, i, j). Importantly, IR induced growth arrest as

90 measured by cell counts for 1 week after IR treatment (Fig. S1k). Furthermore, DAPI intensity

91 has been shown to decrease with senescence ${ }^{19}$ and this was indeed the case in RS and IR 
92 treated cells (Fig. S1I). Using these established senescent models, DAPI-stained nuclei from IR

93 and RS senescent cells were imaged with a high-content microscope. Nuclei were detected

94 using a deep convolutional neural network based on U-net, which produced output images

95 containing the detected nuclear regions. Each detected nucleus was extracted for subsequent

96 analysis. We applied several methods to normalize features in images, such as removing the

97 background, standardizing the size of the nuclei, and even masking inner details of the nuclei

98 (Fig. 1a, b).

99

100

\section{Senescent Cells Display Altered Nuclear Morphology}

102

103

A morphological analysis of the detected nuclei was performed to compare control cells to those

104 that were senescent. There was a significant difference in nuclear area for each of the three groups as previously reported ${ }^{13}$. In addition, IR senescent cells were significantly larger than RS cells (Fig. 1c). Aging and certain premature aging diseases have been associated with

107 greater irregularities or folds in the nuclear envelope ${ }^{20,21}$. We therefore evaluated convexity, 108 which is a ratio of nuclear perimeter to convex hull perimeter, as a measure of the nuclear 109 envelope regularity. Convexity showed the shape of control cells were more regular compared 110 to both IR and RS, which had a more irregular boundary (Fig. 1d). RS had the lowest convexity 111 value, indicating the highest irregularity. This suggests convexity is another measure of 112 senescence, with lower values corresponding to increased senescence. In addition, we looked 113 at aspect ratio, a measure of width compared to height (measured as the longest compared to 114 shortest dimensions of a minimized, rotated rectangle around each nucleus) and found that both 115 IR and RS had higher values compared to control (Fig. 1e). We evaluated area and convexity 116 per nuclei, observing overlapping clusters for the three states with area of RS overlapping both 117 control and IR, and convexity of RS and IR overlapping (Fig. 1f). Interestingly, the distribution of Page 5 of 44 


\section{A DAPI-stained nuclei}
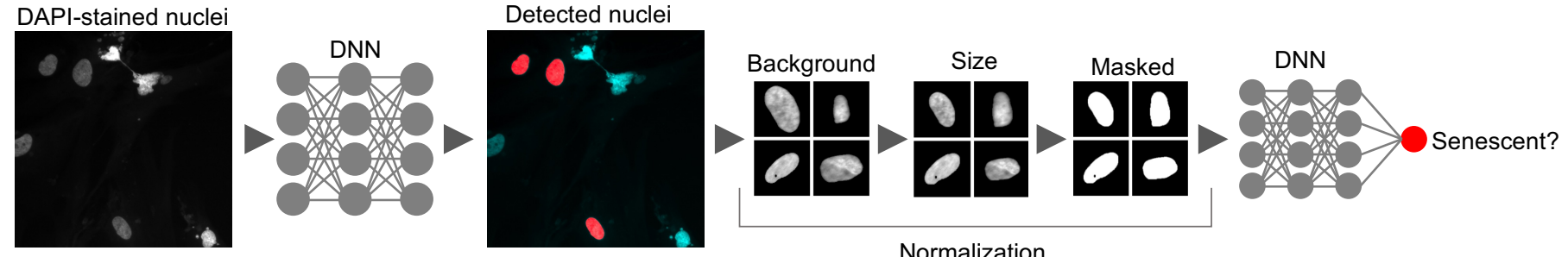

Normalization

B

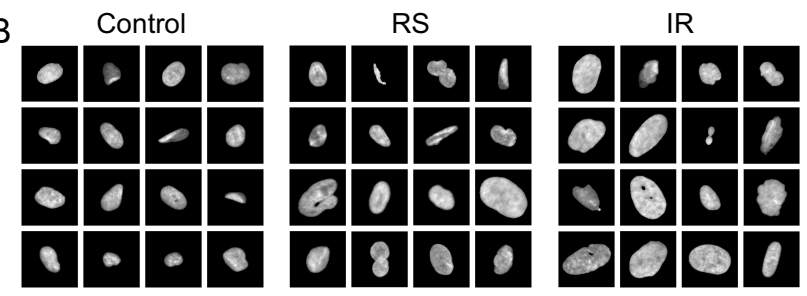

G
$\mathrm{F}$

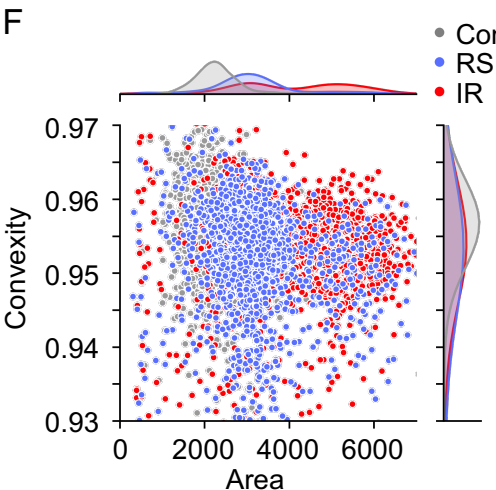

C

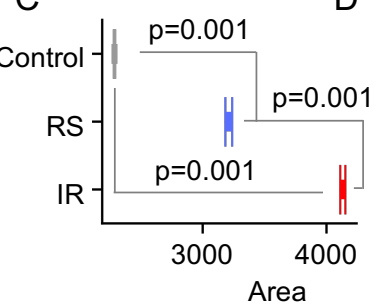

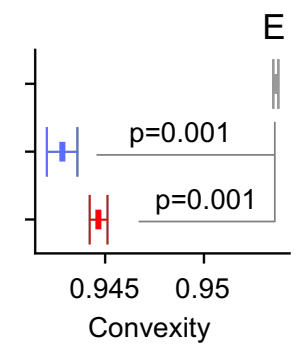

E

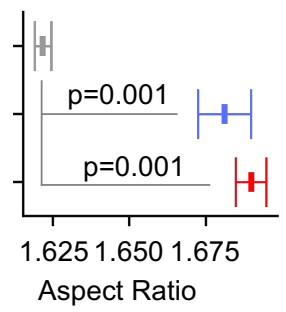

$\mathrm{H}$

I
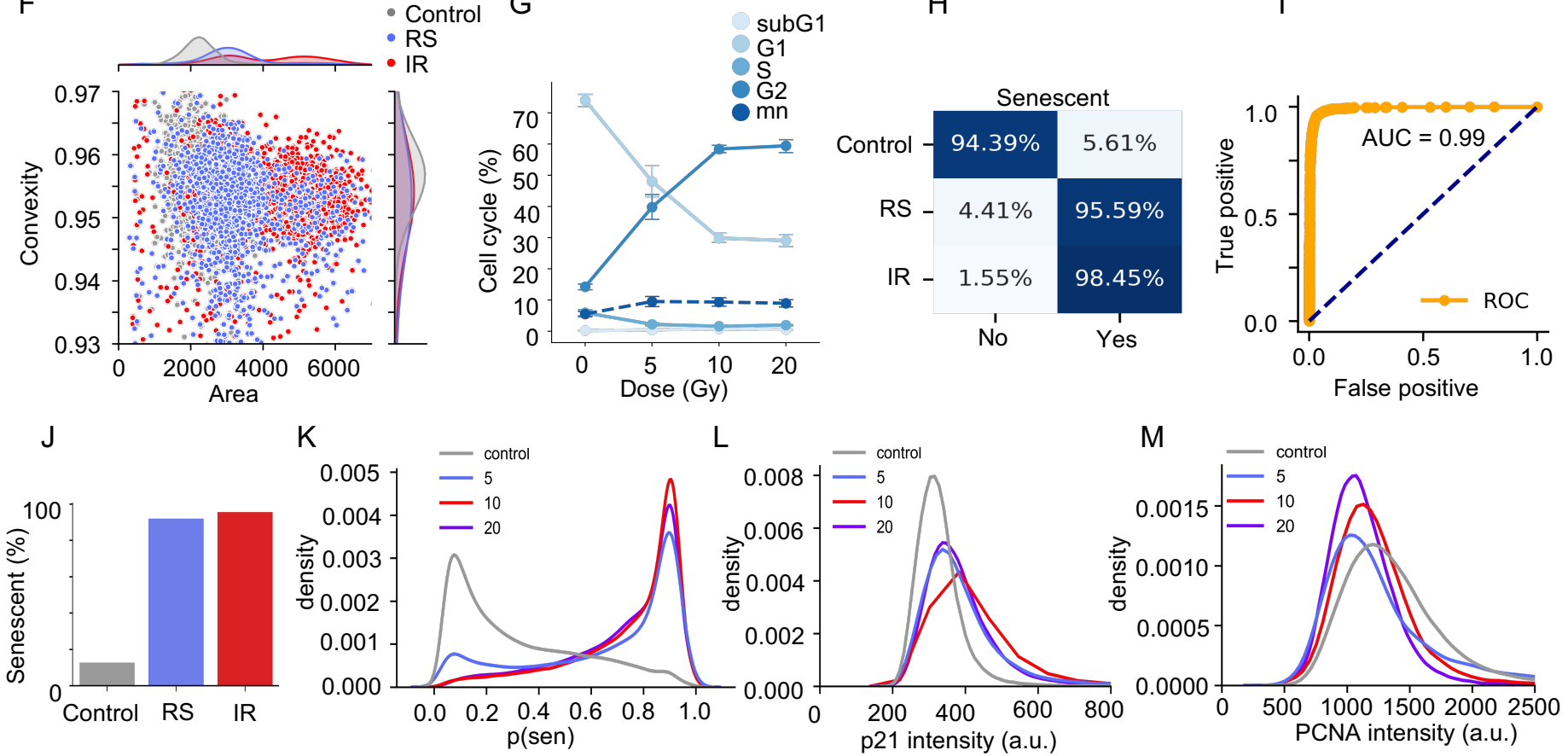

$\mathrm{L}$
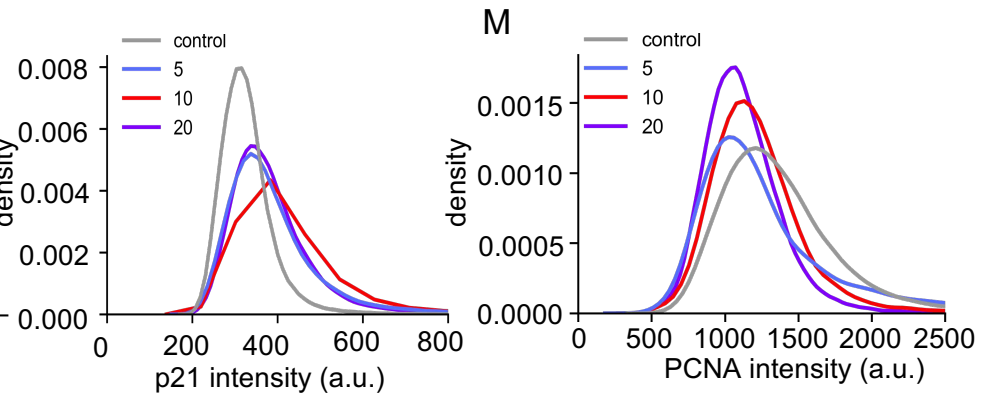

$N$
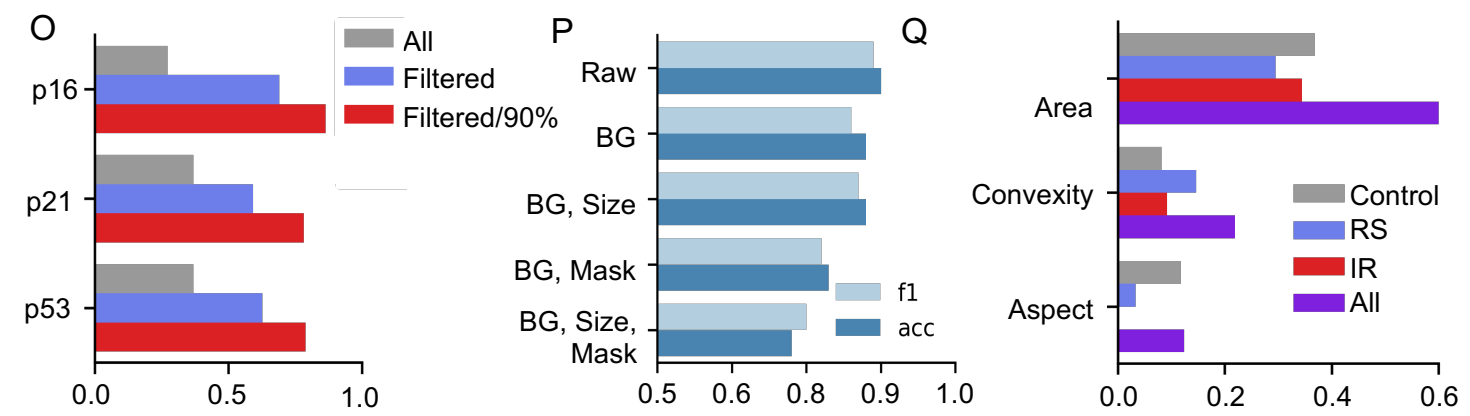

Pearson Correlation Coefficient Pearson Correlation Coefficient

Pearson Correlation Coefficient

Figure 1 Nuclear morphology is an accurate senescence predictor in cultured cells. a Analysis workflow. b Sample nuclei for controls, replicative senescence (RS) and ionizing radiation (IR) induced senescent cells. c Area of identified nuclei $(\mathrm{n}=6,976-68,971$, mean $\pm 95 \% \mathrm{Cl})$. d Convexity of identified nuclei $(n=6,976-68,971$, mean $\pm 95 \% \mathrm{Cl})$. e Aspect ratio of identified nuclei $(n=6,976-68,971$, mean $\pm 95 \% \mathrm{Cl})$. $\mathrm{f} \mathrm{Scatter} \mathrm{plot}$ of individual nuclei, with overall distributions for each at the top and right margins. $g$ Cell cycle analysis after exposure to several doses of IR; mn: multinucleated cells. $\mathbf{h}$ Accuracy of a deep neural network (DNN) predictor on validation data. i Receiver operating characteristics (ROC) curve of the DNN. $\mathbf{j}$ Percent of nuclei in each state classified as senescent for independent cell lines. $\mathbf{k}$ Distribution of prediction probabilities for several doses of IR for three fibroblast cell lines ( $n=38,284-106,132)$. I Distribution of $p 21$ intensities for several doses of IR for three fibroblast cell lines $(n=38,284-106,132)$. $m$ Distribution of PCNA intensities for several doses of IR for three fibroblast cell lines ( $n=38,284-106,132)$. $\mathbf{n}$ Correlation between predicted senescence and nearby SA- $\beta$-gal regions, showing all and $90 \%$ confidence predictions only for RS and IR groups. o Correlation between predicted senescence and multiple markers, showing all, filtered for markers with strong signals, and filtered with $90 \%$ confidence predictions only. $\mathbf{p}$ Accuracy of DNNs trained and predicting after different normalization methods. $\mathbf{q}$ Correlation between morphological metrics and predicted senescence by class, BG: background. 
118 the area of the IR senescent cells was bimodal, with the lower mode matching RS and a higher

119 mode at almost twice the area of the RS, perhaps suggesting IR induced aneuploidy or stalling

120 at the G2 checkpoint of the cell cycle (Fig. 1f, upper histogram distribution of joint scatter plot).

121 To further explore this hypothesis, we induced senescence with multiple IR doses and utilized

122 flow cytometry to study the cell cycle. Remarkably, we observed a dose-dependent increase in

123 G2 and corresponding loss of G1 and S-phase cells 10 days after the IR treatment (Fig. 1g),

124 indicating that IR induction leads to G2 stalled senescent cells as previously suggested ${ }^{22,23}$.

125 Simple nuclear morphological measures appear to be a viable method for assessing cellular

126 senescence in culture.

Deep Learning Classifiers Accurately Predicts Senescence Based on DAPI staining

131 Given the rich structure of nuclei and potentially broad set of features, we applied deep neural

132 networks to better assess senescence. A custom convolutional neural network was trained

133 using $80 \%$ of the samples and $20 \%$ was held out for validation. After seeing accuracy converge

134 to a steady level, the model was applied to validation data. We also compared our custom

135 network to Xception, one of the top performing models for image classification that has been

136 often applied to biomedical classification ${ }^{24,25}$. Xception achieved superior results with an f1-

137 score of $94 \%$, accuracy of $95 \%$, and AUC of 0.99 with validation data (Fig. 1 h, i). To eliminate

138 any potential overfitting on the experimental context and cell lines, we evaluated the model on

139 an independent data set of two additional cell lines, which were prepared and imaged

140 separately. This achieved an f1-score of $92 \%$, accuracy of $94 \%$, and AUC of 0.96 (Fig. S2a, b,

141 c). The predictor identifies senescence for $12.7 \%$ of control, $92.0 \%$ of RS, and $95.6 \%$ for IR

142 (Fig. 1j).

143

Page 6 of 44 
144 To better characterize the performance with senescent phenotypes induced by multiple levels of

145 stress, we applied the DNN predictor to cells exposed to different doses of radiation. All levels

146 were predicted to be senescent, and there was a $9.7 \%$ mean increase between 5 and $10 \mathrm{~Gy}$,

147 but 10 Gy to 20 Gy show a similar prediction score (Fig. 1k). PCNA declines with increasing

148 dose and p21 increases (Fig. 1I, m). Predicted senescence and the two markers align with

149 experimental conditions, but the predictor appears to track p21 expression more closely than

150 PCNA. This experiment indicates that the treatment dose influences the senescent phenotype

151 up to $10 \mathrm{~Gy}$, a dose commonly used for senescence induction.

152

153 In another experiment, a deep neural network was trained to detect control compared to

154 different senescent types, IR and RS. Xception, trained like the dual state model above,

155 produced a mean class accuracy of $78.6 \%$ in detection of the three states, with $83.3 \%$ for

156 controls, $75.7 \%$ for RS, and $76.8 \%$ for IR (Fig. S2d, e). It achieved a relatively high AUC of 0.9

157 for RS and 0.95 for IR. In sum, nuclear morphology represents a strong predictor of both

158 replicative and DNA damage induced senescence. 
164 To confirm the accuracy of the DNN, we evaluated the correlation between predictions and 165 traditional markers of senescence, including SA- $\beta$-gal, p16, p21, and p53. Training a deep 166 neural network to recognize SA- $\beta$-gal regions, we found SA- $\beta$-gal near nuclei for $64.1 \%$ of IR 167 and $65.8 \%$ of RS compared to $19.6 \%$ for control, which roughly matches published rates for RS 168 and controls ${ }^{26}$. A correlation analysis revealed a Pearson coefficient of 0.39 for IR and 0.31 for 169 RS between predicted senescence and SA- $\beta$-gal detected nearby, but when restricting to the 170 treated cells with nearby SA- $\beta$-gal and controls without it, the correlation rose to 0.83 for IR and 1710.67 for RS (Fig. 1n). Applying a 90\% confidence filter (see sections below on confidence and 172 deep ensemble methods), correlation rose to 0.96 for IR and 0.90 for RS, indicating the 173 predictor is highly effective at recognizing senescence with detected SA- $\beta$-gal. On a per cell 174 basis, we found moderate correlation between $\mathrm{p} 16, \mathrm{p} 21$, and $\mathrm{p} 53$ stain intensities and predicted 175 senescence, but after applying a threshold to classify as positive markers and filtering out nuclei 176 near the threshold (due to the broad overlap in the distribution of intensities, Fig. S1g, h, i), the 177 correlation rose significantly to 0.69 for $p 16,0.59$ for $p 21$, and 0.63 for $p 53$ (Fig. 1o). We also 178 applied confidence filtering, restricting nuclei to those with high predictive confidence, and found 179 correlation of 0.86 for $\mathrm{p} 16,0.78$ for $\mathrm{p} 21$, and 0.79 for $\mathrm{p} 53$, indicating high confidence predictions 180 are effectively identifying senescent nuclei with $\mathrm{p} 16, \mathrm{p} 21$, and $\mathrm{p} 53$. The deep predictor is 181 inferring senescence in agreement with multiple markers of senescence. 
187 Nuclei images contain several features that could be used for classification; however, it is 188 unclear what the deep neural network is using as its basis for assessment. Nuclear area, 189 staining intensity and even the image background itself could contain a signal that the neural 190 network is picking up on. To provide some insight into how much these potential factors 191 contribute to senescence classification, we trained several models based on reduced forms of 192 the cutout library. Our base model already includes brightness standardization. First, the 193 background of the nuclei was masked, by excluding all areas outside of the U-Net detected 194 nuclear region. Next, we applied size normalization, such that the greater of the width and 195 height was set to a standard pixel size. Finally, we converted the interior of nuclei to a single196 color value, essentially masking all internal structure. With each reduction, we observed a slight 197 decrease in classification accuracy when applied to independent test lines (Fig.1p). The 198 background masking produced $86 \%$ for the $\mathrm{f} 1$-score and $88 \%$ for accuracy, a small reduction 199 indicating limited reliance of the background. With background masked and size normalized, a 200 trained model produced $87 \%$ for $\mathrm{f} 1$-score and $88 \%$ for accuracy, showing area and size played 201 little role in senescent detection. This model was further reduced by completely masking the 202 internal structure of the nuclei, which led to an f1-score of $80 \%$ and accuracy of $78 \%$ (Fig. S2f, 203 g). While masking was a significant reduction in accuracy, it is remarkable that so much 204 information could be removed from nuclear images and still obtain a relatively accurate 205 classification of senescence. These experiments suggest that classification is largely based on 206 the overall shape of the nuclei. We explored this further by evaluating Pearson correlation 207 between predicted senescence and several morphological metrics, finding that area was 208 moderately correlated (despite being standardized by the predictor) but convexity and aspect 209 ratio were weaker (Fig. 1q). The deep learning model appears to be picking up on the nuclear 210 shape in a more sophisticated manner than simple morphometrics. 
212 The final reduced model yields an overall accuracy of $78 \%$, and it shows an imbalanced per

213 class accuracy of $73.9 \%$ for control, $69.3 \%$ for RS, and $91.4 \%$ for IR. It maintains a good AUC

214 of 0.88 . With similar reductions, the three-state senescent type detector model shows overall

215 accuracy of only $58 \%$ (Fig. S2h, i): $87.7 \%$ for controls, $56.1 \%$ for IR, but only $31.3 \%$ for RS. The

216 AUC has declined to 0.71 for RS and 0.6 for IR. Despite lowering accuracy, the feature

217 standardization and reduction makes the model less influenced by a large number of technical

218 variations such as image intensity, choice of nuclear staining method, magnification and others

219 that could impact the utility of the predictor.

\section{Classification with Confidence}

224 While overall accuracy per-nuclei was relatively high, a sizable number of nuclear images were

225 ambiguous, which can be interpreted as the model being uncertain in its prediction. Extending 226 neural networks with Bayesian properties has several advantages, most notably providing a 227 measure of confidence for predictions ${ }^{27}$. The Bayesian Neural Networks (BNN) allows for the 228 construction of a posterior probability distribution which can be used for interval estimation, 229 compared to a single prediction from a classic neural network. Samples can be filtered to 230 reduce the ambiguous cases by requiring higher mean probability from the BNN. Using 231 Tensorflow Probability, we developed several BNNs. Our custom model converted to a BNN 232 performed adequately for raw cutouts, but it would not train well for the masked/normalized 233 nuclei. We partially converted Xception to utilize Flipout nodes ${ }^{28}$, leaving the separable 234 convolutions as point estimate nodes. We also fully converted InceptionV3 as an alternative 235 model. Our partial BNN of Xception produced an f1-score of $84 \%$, accuracy of $86 \%$, and AUC of 2360.92 (Fig. S2j, k). The full BNN for InceptionV3 gave an f1-score of $79 \%$, accuracy of $80 \%$, and Page 10 of 44 
237 AUC of 0.87 (Fig. S2I, $\mathbf{m}$ ). The BNN models can thus be used to understand the probability

238 distribution of the data but at a lower accuracy.

\section{A Deep Neural Network Ensemble Increases Predictive Power}

243 After training the senescent classifier through different sessions, we saw variance in the

244 predictions for a subset of samples. Exploring a large multidimensional solution space during

245 training, neural networks select a relatively good solution that is often biased to favor certain

246 classes ${ }^{29}$. Using an ensemble of deep models, the predictions can be combined as though

247 consulting a collection of experts (or interpreted as the "wisdom of the crowd"). To achieve this,

248 we trained an ensemble with random initial weights, potentially allowing convergence to different

249 local minima. We found that there is consistent agreement for the majority of samples, however,

250 there is a significant percent of edge cases with a high variance in predictions among the model 251 instances (Fig. 2a).

253 We therefore speculate that using an ensemble of deep models for inference and aggregating 254 the results provides predictions with less bias and higher confidence (Fig. 2b). Evidently, some 255 models balance the accuracy of each class in the middle of the range $(75-80 \%)$, while other 256 models skew toward one class at the expense of the other (for example, obtaining $\sim 85 \%$ on one 257 but $\sim 70 \%$ on the other). While ensembles have benefits like a BNN, they can be less biased 258 since each ensemble member can specialize around a solution, while a BNN is confined to a 259 single local minima in solution space. Accordingly, we obtained good results with the ensemble 260 method, with an f1-score of $91 \%$, accuracy of $94 \%$, AUC of 0.98 (Fig. 2c, d). More importantly, 261 the ensemble provides a higher confidence and less biased approach by combining multiple 262 models that specialize in predicting different classes. 


\section{An ensemble of neural networks outperforms Bayesian neural networks}

We also tried Bagging, where bootstrapping with replacement selects a subset of the samples to use in training independent models. This method did not provide a significant improvement

267 over the basic deep ensemble method (Fig. 2e). The BNN models can be used to improve 268 confidence but sacrifice performance, while the ensemble models provide both (Fig. 2e). We 269 therefore further evaluated the deep ensemble method with masked and normalized samples.

270 This produced an f1-score of $80 \%$, accuracy of $82 \%$, and AUC of 0.89 (Fig. 2f, $\mathbf{g}$ ), which

271 improved upon the single model. The ensemble method was also applied to the tri-state model 272 to distinguish senescent type, which achieved overall accuracy of $66 \%$ and AUC of 0.81 for RS 273 and 0.92 for IR (Fig. 2h, i). While this is lower accuracy, it is an overall improvement of $23.64 \%$ 274 compared to the single normalized tri-state model. With all states well above the $33.3 \%$ 275 accuracy expected from random predictions, this model is capable of recognizing type of 276 senescence given an adequate sample size.

278 Due to the lower performance of senescent type prediction, we trained deep models on each 279 type of senescence exclusively, training for control vs RS-only and control vs IR-only. This 280 leaves the other state undefined, assessing each type of senescence separately. Both models 281 classified IR with high accuracy, but the RS-only model recognized RS with $\sim 13 \%$ higher 282 accuracy, while the IR-only misclassified those as control (Fig. 2j, k). Ensembles of deep neural 283 networks clearly allow for greater accuracy for senescence prediction. 
Deep neural networks utilizing one-hot node outputs with the softmax function are trained to produce numerical values that are sometimes treated as the probability for each state. They should not be interpreted as model confidence, but by sampling from a BNN or deep ensemble, we can utilize the distribution to determine uncertainty ${ }^{27}$. We evaluated the predictions for the

293 BNN and deep ensemble (Fig. S3a, b). Correct predictions are indeed oriented toward the 294 lower and higher range of the softmax output, representing greater certainty about a sample's 295 state. In both cases, the incorrect predictions are clustered toward the center near the 0.5 296 threshold. Different models could be biased toward either state by shifting those ambiguous 297 samples across the threshold.

We can assume higher confidence in a model's predictions by raising the classification threshold (of both one-hot states, thereby filtering the predictions in the middle). We therefore evaluated the accuracy using a range of thresholds from 0.5 up to 0.95 in the single model, the Xception BNN, the ensemble of models, and the ensemble of fully normalized models (Fig. S3c, d, e, f). In all cases, we see a significant increase in accuracy as the threshold is raised, due to 304 the ambiguous samples being discarded. By raising the threshold, the Xception-based BNN goes from $85.6 \%$ to $96.0 \%$, while the ensemble of normalized models goes from $81.6 \%$ accuracy to $97.2 \%$. A similar approach was applied to other models, including the IR-only and

307 RS-only models (Fig. S3g, h). Raising the threshold, these also showed a gain in accuracy of $30810-15 \%$. Unfortunately, this led to a significant reduction in the number of samples considered.

309 There is a tradeoff between number of predictions and accuracy, which must be balanced for 310 each application to ensure suitable power for analysis. 
315 To better understand the development of the senescent phenotype and how nuclear

316 morphology changes over time, we analyzed fibroblasts induced to senescence by 10 Gys of IR

317 and imaged at several time points, including 10, 17, 24, and 31 days. The predictor identifies

318 senescence at all four times points with probability that increases from days 10 to 17 but

319 declines by day 31 (Fig. S4a). Interestingly, examining the probability distribution of the

320 predictor it was apparent that a growing peak of non-senescent cells appear after day 17, either

321 suggesting that the predictor is unable to accurately predict those cells or that a small number of

322 cells may have escaped senescence and are eventually overgrowing the non-senescent cells

323 (Fig. S4b). Indeed, when investigating markers of proliferation, we see that over the time

324 course, PCNA declines until day 17 after which the expression starts to return (Fig. S4c). p21

325 follows an inverse pattern with stain intensity increasing initially and then declining slightly by

326 day 31 (Fig. S4d). We also saw a decrease in DAPI intensity for days 10 and 17, indicating

327 senescence, but a reversion to control level by day 31 (Fig. S4e). To confirm that the predictor

328 accurately determined senescence even 31 days after IR, we evaluated if markers of

329 proliferation and senescence correlated with predicted senescence. Accordingly, cells with

330 predicted senescence had higher p21 levels, lower PCNA and lower DAPI intensities and vice

331 versa (Fig. S4f, g, h). Morphologically, area and aspect are higher for predicted senescence

332 while convexity is lower (Fig. S4i, j, k). Finally, a simple nuclei count confirms growth, following

333 IR treatment (Fig. S4I). Overall, the senescence predictor captures the state during

334 development in agreement with multiple markers and morphological signs. 
340 Senescent cells are associated with the appearance of persistent nuclear foci of the DNA

341 damage markers $\gamma \mathrm{H} 2 \mathrm{AX}$ and 53BP ${ }^{30,31}$. We characterized the DNA damage foci for our cell

342 lines and investigated how these foci relate to predicted senescence. Our base data set

343 including control, RS, and IR lines were examined for damage foci. Using high content

344 microscopy, we counted DNA damage foci per nuclei and found the mean count of $\gamma \mathrm{H} 2 \mathrm{AX}$ and

345 53BP1 foci to be below 1 each ( 0.9 and 0.6 , respectively) for controls, while RS had $4.0 \gamma \mathrm{H} 2 \mathrm{AX}$

346 and 2.0 53BP1 foci and IR had 3.4 H $\gamma 2 \mathrm{AX}$ and 3.0 53BP1 foci (Fig. 3a, b, S5a). To study how

347 the presence of damage foci relates to predicted senescence, we calculated the Pearson

348 Correlation between predicted senescence and $\gamma \mathrm{H} 2 \mathrm{AX}$ and 53BP1 foci counts. We found that

349 across all conditions there is a moderately strong correlation of around 0.5 (Fig. 3c). This

350 association is also visible when simply plotting foci counts and senescence prediction which

351 shows predicted senescence flipping from low to high, along with shifts in foci counts (Fig. S5b).

352 Within senescent subtypes RS and IR, the correlation is slightly weaker, perhaps indicating that

353 the senescent probability score for each subtype has some correlation with foci count. Our

354 feature reduction including masking means that internal nuclear structure was not used in

355 assessment, but it is nonetheless notable that senescence prediction (overall and by subtype)

356 correlates with foci count. We also compared the correlation between predicted senescence and

357 area. Here too, we see a correlation of around 0.5 , and slightly weaker for the subtypes. In sum, 358 there is a considerable correlation between foci counts and senescence. 

364 Patients suffering from premature aging, or progeria, represent genetically well-defined models

365 to understand the molecular basis of aging ${ }^{32,33}$. To test if cell lines from progeria patients

366 display accelerated aging in culture, we applied the senescent classifier to primary fibroblasts

367 isolated from Hutchinson-Gilford progeria syndrome (HGPS), ataxia telangiectasia (AT) and

368 Cockayne syndrome (CS) (Fig. 3d). Evaluating the area of the nuclei of progeria cells, we found

369 that in general their mean is significantly larger than controls. Notably ataxia-telangiectasia cells

370 have the largest nuclei at $25 \%$ higher than controls, while Hutchinson-Gilford progeria and

371 Cockayne syndrome are both 15\% higher (Fig. 3e). We also investigated DNA damage foci and

372 observe that most prematurely aged lines have higher $\gamma \mathrm{H} 2 \mathrm{AX}$ and 53BP1 foci counts (Fig. 3f, g,

373 S5c). Further, despite diverse mechanisms, the classifier recognized these cell lines having

374 significantly greater probability of senescence (Fig. 3h). All progeria lines have high mean

375 probability of senescence at 0.7 , indicating that the average cell in each group is considered

376 senescent, while controls are below the standard threshold at 0.3 . Evaluating SA- $\beta$-gal activity,

377 we find $35-60 \%$ of nuclei have positivity and overall correlation of 0.5 between predicted

378 senescence and having nearby SA- $\beta$-gal(Fig. 3i). When predictions are filtered to higher

379 confidence levels, there is an increase in correlation up to 0.9 (Fig. 3j), indicating high

380 confidence predictions are capturing the senescent state. DAPI intensity also suggests that all

381 progeria lines have higher senescence compared to controls (Fig. 3k). These observations

382 indicate that our classifier may be able to discriminate rates of aging in cultured cells. 
388 To broaden the applicability of our classifier we speculated that it might apply to nuclei from 389 other cell lines and species. We therefore evaluated the model on mouse primary astrocytes 390 and neurons treated with IR (Fig. 3I). While astrocytes are known to senesce with cell cycle 391 arrest, post-mitotic neurons also exhibit a senescence-like state ${ }^{34}$. We first compared the nuclei

392 area and found that the IR-treated astrocytes had slightly but significantly larger nuclei than 393 controls while IR-treated neurons had reduced area, unlike other cell types we studied (Fig. 3m,

394 n). Evaluating DNA damage foci, we see that IR treated astrocytes and neurons have 395 substantially higher foci count as expected (Fig. 3o, p, q, r). We next applied the ensemble of 396 deep models and found that the IR treated astrocytes had a $7.7 \%$ higher probability of 397 senescence than controls while IR-treated neurons have $6.3 \%$ higher probability (Fig. $3 \mathbf{s , ~ t}$ ).

\section{Senescence prediction translates across species and tissues in vivo}

We applied the predictor to H\&E-stained liver tissue from C57BI6 mice at taken at 48, 58, and 78 weeks of age. After imaging the tissue sections at 20x, we used a deep learning segmentation model trained on 18 tiles to extract nuclei from 16,187 tiles (Fig. 4a). Our training set included samples of hepatocytes only, and this cell type was primarily selected during automated segmentation. We first analyzed morphological metrics, finding an insignificant

407 increase in nuclear area (Fig. 4b). However, we saw a significant decrease in convexity and 408 increase in aspect ratio, both indicating increased senescence with age (Fig. 4c, d). Nuclei 409 were evaluated for senescence using the normalized RS-only and IR-only models, of which the 410 RS model indicated increasing senescence with age while the IR model did not (Fig. 4e, f).

411 Using the probability, we calculated the percent of senescent cells, finding $\sim 36 \%$ for RS and Page 17 of 44 
$412 \sim 99 \%$ for IR. The predictor is trained on DAPI-stained cultured fibroblasts representing a

413 considerable difference in context, it is therefore likely that the algorithm should be tuned to

414 evaluate other data sources. Applying thresholds of 0.6 and 0.9 for RS and IR, respectively, the

415 percent was brought down to roughly 8-10\% to match the percent reported, roughly adjusted for

416 difference in age and split between IR and RS ${ }^{35}$. With these thresholds, the percent of

417 senescent cells per mouse increased with age (Fig. $\mathbf{4 g}, \mathbf{h}$ ). To determine the predictor's ability

418 to identify senescent hepatocytes in liver tissue, we also stained tissue sections from the same

419 specimens with DAPI and p21, identified hepatocytes with segmentation, and predicted

420 senescence of those nuclei. We found that the mean predicted senescence per animal for the

421 p21+ cells was significantly higher compared to p21- for both RS and IR models (Fig. 4i, j).

422 Given the differences in human and mouse nuclei as well as between cell types, it is notable

423 that the senescent state can be captured through the relative difference in assessed probability.

424 It therefore appears that our predictor may be able to determine senescence across cell types

425 and species.

\section{Predictor to Recognize Senescence from Multiple Mechanisms}

430 Senescence can be induced by several types of stressors that could result in different types of 431 pathologies ${ }^{36-38}$. We therefore induced senescence by different drug treatments, including the

432 DNA damaging compound doxorubicin, the mitochondrial toxin antimycin A, and the HIV

433 protease inhibitor atazanavir/ritonavir (ATV/r) and evaluated predictors for each of them. PCNA

434 staining confirmed that each drug treatment led to a senescent state (Fig. 4k). A morphometric 435 analysis showed that all three drug treatments expanded nuclear area, decreased convexity,

436 and increased aspect ratio (Fig. 4I, m, n). Additionally, DAPI intensity decreased significantly for 437 all three treatments, indicating senescence (Fig. 40). The predictor model (trained on IR and RS Page 18 of 44 
438 methods) recognized senescence in the nuclei treated with doxorubicin but did not detect

439 senescence in treatment with antimycin A or ATV/r (Fig. 4p). We speculate that doxorubicin

440 treatment more closely resembles the DNA damage caused by IR-induced and replicative

441 senescence. To address this limitation of our model, we trained new models for each new type,

442 including doxorubicin-only, Antimycin A-only, and ATV/r-only (Fig. 4q, r, s). In addition, we

443 trained on a broader data set, including IR, RS, doxorubicin, antimycin A, and ATV/r. Tested on

444 validation data held out from training, we find the expanded model can now recognize antimycin

445 A with $66.0 \%$ accuracy, ATV/r with 64.3\% accuracy, and doxorubicin with $62.3 \%$ accuracy,

446 which exceeds performance for each individual predictor (Fig. 4t). However, it has reduced

447 accuracy for IR at $68.1 \%$ (compared to $83.0 \%$ ), although RS is slightly higher in this model.

448 While the base predictor model provides higher accuracy for IR, the unified model can

449 recognize senescence in more diverse conditions.

\section{Predictor Detects Senescence in Human Dermal Tissue with p21}

454 To determine if the predictor could be used with human dermis, we analyzed samples from an 455 independent data set ${ }^{39}$, stained with hematoxylin and DAB for p21 (Fig. S6a). Nuclei were 456 detected using image segmentation with U-NET trained on the hematoxylin nuclei, and the 457 predictor generated senescence probability scores for the extracted nuclei. After calibrating the 458 p21 detection threshold to roughly match published rates, we found the mean predicted 459 senescence of $\mathrm{p} 21+$ nuclei was $5.9 \%$ higher than those without $\mathrm{p} 21$ for the RS and IR models 460 (with $p=0.005$ for both), while other models showed no difference (Fig. 5a, S6b, c, d, e). As the 461 confidence threshold was raised above the standard 0.5, p21+ was clearly separated from p21-. 462 With increasing confidence, the p21+ nuclei generally showed higher predicted probability for 463 IR, while the p21- nuclei showed lower predicted probability for RS. The percent difference Page 19 of 44 
464 between mean p21+ and p21- probability also increased with higher confidence. Notably, all

465 three other models (for doxorubicin, ATV/r, and antimycin-A) showed no separation between

466 p21+ and p21-, indicating that they are picking up on other type-specific aspects of senescence.

The human dermis shows age-dependent increase in senescent nuclei

471 To further investigate if the predictor could be applied in a clinical context, we tested the

472 algorithm on human skin samples of 169 individuals aged 20-86 years. The senescent classifier

473 was used to evaluate the dermal nuclei from biopsy samples, stained with H\&E and imaged in a

474 slide scanner at 20x. We used U-Net to detect nuclei, extracted nuclear regions, and converted

475 the nuclei to the normalized and masked form (Fig. 5b). We first evaluated several

476 morphological metrics, including area, convexity, and aspect ratio. Across age, we see no

477 change in area (Fig. 5c), an insignificant change in convexity (Fig. 5d), and a significant change

478 in aspect ratio (Fig. 5e). We considered that different pathologies could be related to various

479 forms of senescence (senescence caused by diverse mechanisms such as DNA damage,

480 telomere attrition, mitochondrial dysfunction, and so on), so we evaluated multiple senescence

481 predictor models developed here. We found the probability of senescence increases with age of

482 patients for RS but is relatively flat for IR and declines for ATV/r, antimycin-A and doxorubicin

483 (Figures S6f, $\mathbf{g}, \mathbf{h}, \mathbf{i}, \mathbf{j}$ ). We expect a percent of human dermal nuclei to be senescent, ranging

484 from $\sim 1 \%$ in young to $\sim 15 \%$ in old ${ }^{39}$, so we selected thresholds to calibrate the model with 0.7

485 for RS and 0.85 for IR, leading to an overall predicted percent of $\sim 6 \%$ and showing an age-

486 dependent increase in percent of senescence (Fig. 5f, $\mathbf{g}$ ). Both IR and RS models predict a

487 statistically significant increase with age, while doxorubin, Antimycin-A, and ATV/r appear

488 decoupled from age (Fig. S6k, I, m). We also evaluated the correlation between morphological

489 metrics and predicted senescence and found moderate correlation for several metrics, but RS

Page 20 of 44 
was more correlated with convexity while IR was more correlated with area and aspect ratio,

491 perhaps indicating morphological aspects of each type of senescence in vivo (Fig. S6n, S7a).

492 Interestingly, we found that area was anti-correlated with both predicted IR and RS, but

493 predicted IR was inverse to aspect ratio. This indicates difference between senescence in

494 culture and in tissue sections and affirms that the IR and RS model are picking up on different

495 aspects of senescence. We considered whether the age-dependent increase in predicted

496 senescence could be related to change in proportions of detected cell types, so we compared

497 the distribution of cell area and aspect ratio for broad age groups, individuals below 40 and

498 those over 60. A shift in cell types should be reflected by a change in these metrics, but we

499 found no noticeable difference in the distribution of these metrics for predicted senescent and

500 non-senescent cells between age groups (Fig. S7b, C). Comparing each group by mean area

501 and aspect ratio of individuals, a t-test shows non-significance $(p=0.94$ and $p=0.51$,

502 respectively), indicating that each group has a similar proportion of cell types.

503

504

Senescent dermal nuclei are inversely associated with neoplasms and positively correlated with hypertension and osteoporosis

507

508 Given the large variation in predicted senescence, we speculated that these values could 509 represent meaningful health outcomes. To investigate, we retrieved 19,820 ICD-10 diagnosis 510 codes collected in the Danish National Patient Register from 1977 to 2018 for all the individuals

511 in the study. We looked for associations between individuals with diagnosed conditions grouped 512 by ICD-10 chapters and predicted senescence above or below the age-dependent mean (those

513 above or below the trendline in Fig. 5f, $\mathbf{g}$ and S6f-m specifically using residuals from linear

514 regression of predicted senescence versus age), using the chi-squared test and Fisher's exact

515 test for the frequency of occurrence between the two groups (Fig. 5h-m). Remarkably, we found Page 21 of 44 
516 a significant correlation between a rate of senescence below the age-matched mean and the

517 presence of ICD-10 Chapter II Neoplasm diagnosis codes for both RS and IR, with p-values of

5180.002 and 0.005 , respectively (Fig. 5n). Narrowing down the analysis we determined the

519 association was based on malignant (versus benign or unknown) codes within ICD-10 Chapter

520 II Neoplasm with IR p-value at 0.018 and RS at 0.058 . Notably, RS better represents replicative

521 senescence which occurs naturally with age, while IR better represents DNA damage, although

522 there is considerable overlap in predictions between the two with this model. We also scanned

523 individual ICD-10 clinical codes and found several other conditions associated with senescence,

524 including osteoporosis, osteoarthritis, hypertension, cerebral infarction, hyperlipidemia,

525 hypercholesteremia, and hearing loss, which were all significant when evaluated individually but

526 non-significant when applying multiple test correction, such as the Bonferroni (Fig. 5n, S7d-o,

527 S8a). All of these conditions were associated with higher levels of predicted dermal senescence

528 except for cancer and hearing loss, which were associated with lower levels of predicted

529 senescence. They draw from different models, for example neoplasms are particularly

530 significant with RS, while hypertension only appear in the Antimycin-A model. Overall, we found

531 that high assessed senescence corresponds to fewer neoplasms and malignancies, while also

532 indicating increased frequency of osteoporosis, osteoarthritis, hypertension, and other

533 conditions.

534

535

536

Page 22 of 44 


\section{Discussion}

539 In this paper we present a neural network classifier that can predict cellular senescence based

540 on nuclear morphology. Trained on fibroblasts maintained in cell culture, the classifier achieves

541 very accurate results, which was confirmed by applying it to independent cell lines. We also

542 trained models to correctly distinguish between senescence caused by radiation induced

543 damage and replicative exhaustion. By training additional models on samples with reduced

544 features, we infer that the shape of the nucleus alone provides a significant signal to indicate

545 senescent state. DAPI-stained nuclei with background removed, size normalized, and internal

546 structure masked are still classified with high accuracy. These feature reduction methods serve

547 a secondary purpose, making a model robust to technical variation - our neural network trained

548 on reduced samples can make predictions on nuclei that were prepared in other experimental

549 and imaging contexts. Indeed, the predictor distinguished senescent astrocytes and neurons,

550 predicted an age-related increase in senescent liver cells, and confirmed senescence in cell

551 lines from patients suffering from premature aging. Although it is still debated if universal

552 markers of senescence exist, our findings suggest that at least morphological alterations in

553 nuclei may be common across some tissues and species.

555 We present several predictor models, including those that combine IR, RS and other methods,

556 and those that specialize on each for improved accuracy. The base model trained on IR and RS

557 can identify either type along with senescence induced by doxorubicin, indicating that the

558 predictor has identified features found in multiple types related to DNA damage. Our base

559 model did not accurately identify ATV/r and antimycin A, but a new model trained on all five

560 methods could accurately identify senescence induced by these diverse mechanisms. The 
561 unified model could be identifying a common signature or simply recognizing multiple

562 phenotypes.

564 Our data shows that individuals with a predicted higher rate of senescent cells have reduced

565 neoplasms and malignant cancer, in comparison to those with a lower rate of senescence.

566 This is highly consistent with the notion that senescence is a likely mechanism to control cancer

567 development by limiting uncontrolled proliferation ${ }^{18}$. Further, premalignant tumors express

568 markers of senescence, which are absent in malignancies, and malignant tumors can regress

569 and undergo senescence by switching off oncogenes ${ }^{17}$, supporting the protective role of

570 senescence in blocking the progression of neoplasms to malignancies. In addition, loss of

571 central senescence inducers such as p16 is very common in many cancer types ${ }^{40}$. Of note,

572 there is also evidence suggesting that cellular senescence promotes malignancy through the

573 inflammatory senescence associated secretory phenotype or SASP ${ }^{41}$, that senescent cells may

574 appear in areas where tumors tend to subsequently develop ${ }^{42}$, and that senescent cells and

575 SASP induced by cancer treatment led to worse survival and healthspan ${ }^{43}$. While the role of

576 senescence in cancer is highly complex, our results based on clinical data support the overall

577 protective role for senescence in human health with regards to cancer. We also found several

578 other conditions often associated with senescence, including osteoporosis, osteoarthritis,

579 hypertension, cerebral infarction, hyperlipidemia, and hypercholesteremia, which appear more

580 frequently in individuals with a higher predicted rate of senescence.

582 We also investigated how our deep learning predictor results correspond to other measures of

583 senescence. Nuclear area is known to expand during senescence ${ }^{13,44,45}$, and we confirmed this

584 fact in our cell culture data set, with significant differences in IR and RS senescent cells. On a

585 per nuclei basis, we found a moderate correlation between area and predicted senescence.

586 However, due to our size normalization, it is unlikely this classic feature is the primary signal for 
587 our deep learning model (at least for the size-normalized version). We also identified convexity

588 and aspect ratio as key morphological properties that differ between control and senescent cells

589 in culture and found moderate correlation between each of these properties and predicted

590 senescence. Interestingly, we found no increase in area with age in the human dermis, but a

591 significant increase in aspect ratio and significant decrease in convexity, indicating nuclei

592 becoming stretched and irregular with advancing age in humans. These observations confirm

593 that size normalization is necessary to generalize our neural network classifier. It also

594 demonstrates the value of our feature-neutral approach, where the neural network is trained to

595 identify senescence from rich image data, and it is later reduced through feature removal.

596

597 In sum, our deep neural network model is capable of accurately predicting the senescent state

598 and type from nuclear morphology using several imaging techniques and has been

599 demonstrated with several diverse applications. We applied the predictor to human skin

600 samples and observed an age-dependent increase in senescence. Remarkably, individuals who

601 appear to have higher rates of senescent cells show reduced incidence of malignant

602 neoplasms. This supports the long-standing hypothesis that senescence is a mechanism to limit

603 cancer. Further, we find association between higher predicted senescent cell burden and other

604 conditions, including osteoporosis, osteoarthritis, hypertension, cerebral infarction,

605 hyperlipidemia, and hypercholesteremia.

606 


\section{Methods}

609 Cell culture

610 All human-derived primary skin fibroblast cells were purchased from Coriell Institute (USA).

611 Control fibroblasts included AG08498 (male, 1 year), GM22159 (male, 1 day), GM22222 (male

6121 day), GM03349 (male, 10 years) and GM05757 (male, 7 years). Cells were cultured at 37C

613 and 5\% CO2 either in 1:1 mix of DMEM GlutaMAX (Gibco, 31966047) and F-12 media (Gibco,

614 31765068) for AG08498, GM22159 and GM22222 or in EMEM media (Biowest, L0415-500) for

615 GM03349 and GM05757. Fibroblasts derived from Hutchinson-Gilford progeria syndrome

616 patients included AG06917 (male, 3 years), AG06297 (male, 8 years) and AG11513 (female, 8

617 years). Fibroblasts sampled from ataxia telangiectasia and Cockayne syndrome patients were

618 GM03395 (male, 13 years) and GM01428 (female, 8 years), correspondingly. Cells were

619 cultured at 37C and 5\% CO2 in MEM media (Lonza, BE12-662F). Freshly isolated primary

620 mouse astrocytes were kindly provided by the Department of Drug Design and Pharmacology,

621 University of Copenhagen. Cells were cultured at 37C and 5\% CO2 in DMEM GlutaMAX (Gibco,

622 31966047). All used media were supplemented with 10\% fetal bovine serum (Sigma-Aldrich,

623 F9665) and $100 \mathrm{U} / \mathrm{mL}$ penicillin-streptomycin (Gibco, 15140163).

624

\section{Senescence induction}

626 To achieve replicative senescence control fibroblasts at early passages were seeded in T25 cell

627 culture flasks (200 000 cells) and cultured over 32 weeks. After each splitting cell number was

628 recorded and population doubling level (PDL) was calculated as $\log _{2}$ (cell number during

629 harvesting/cell number during seeding). Experiment was terminated when PDL reached zero.

630 Induction of cellular senescence by ionizing radiation, doxorubicin, antimycin $\mathrm{A}$ and

631 atazanavir/ritonavir was performed according to ${ }^{46}$. Briefly, control fibroblast cells at early 
632 passages were seeded in 96 well plates (Corning, 3340) in a density of 2000 cells per well. Day

633 after cells either were exposed to 10Gy of ionizing radiation or treated with $250 \mathrm{nM}$ doxorubicin

634 for $24 \mathrm{~h}$ and cultured for the next nine days. Medium was replaced every two days. Three days

635 before radiated or doxorubicin-treated cells reached senescence state, fibroblast cells from the

636 same stock were seeded (2 000 cells/well) as mock-irradiated or DMSO-treated controls.

637 Mitochondrial dysfunction-induced senescence was achieved by treating control fibroblast cells

638 with $250 \mathrm{nM}$ antimycin A every two days within ten days. $25 \mu \mathrm{M}$ atazanavir/ritonavir was given

639 to control fibroblast cells every two days within fourteen days to develop senescence

640 phenotype. Corresponding DMSO-treated controls were cultured in parallel and seeded in 96-

641 well plate three days before terminating the experiment..

642

\section{Gene expression analysis}

644 Radiated and control fibroblast cells were lysed using TRIzol reagent (Ambion by Life

645 technologies) and RNA was isolated using Direct-zol RNA miniprep Plus (Zymo Research).

646 RNA was reverse transcribed into cDNA using High-capacity cDNA reverse transcription kit

647 (Applied Biosciences) according to manufacturer's protocols. Next, RT-qPCR was performed to

648 detect mRNA levels of senescence markers using StepOnePlus Real-Time PCR System

649 (Applied Biosystems). Relative gene expression was calculated based on obtained Ct values,

650 normalized to housekeeping gene GAPDH, and expressed as fold changes compared to

651 non-irradiated control. The following specific primer sequences were used ${ }^{46}$ :

652 CDKN2A (p16):

653 Forward: GAG CAG CAT GGA GCC TTC

654 Reverse: CGT AAC TAT TCG GTG CGT TG

655 CDKN1A (p21):

656 Forward: TCA CTG TCT TGT ACC CTT GTG C

Page 27 of 44 
658 IL6:

659 Forward: CAG GAG CCC AGC TAT GAA CT

660 Reverse: GAA GGC AGC AGG CAA CAC

661 GAPDH:

662 Forward: GTC AGC CGC ATC TTC TTT TG

663 Reverse: GCG CCC AAT ACG ACC AAA TC

664

665 In total three independent sets were performed in GM22159 cells.

666

667 Immunocytochemistry, SA- $\beta$-galactosidase detection and image preparation

668 For detection of persistent DNA damage foci as well as fluorescence intensity levels of $p 16$,

669 p21, p53 and PCNA, fibroblast cells were washed once with warm PBS, fixed in 4\%

670 paraformaldehyde (PFA) for $15 \mathrm{~min}$ followed by permeabilization step with incubation for $10 \mathrm{~min}$

671 in PBS-0.1\% Triton X100. Blocking was performed in 1\% BSA-PBS- $0.1 \%$ Tween 20 overnight at

672 4C. Next day cells were incubated with primary antibodies $(\gamma \mathrm{H} 2 \mathrm{AX}, 1: 1000$, Millipore, 05-636

673 and 53BP1, 1:2000, Novus, NB100-304; p16 ${ }^{\text {INK4A }}, 1: 50$, Santa Cruz, sc-56330; p21, 1:200,

674 Santa Cruz, sc-6246; p53, 1:200, Santa Cruz, sc-126; PCNA, 1:500, Abcam, ab18197) for 1h at

675 RT, washed three times with PBST and incubated with secondary antibodies (1:200 Alexa-Flour 676488 , Invitrogen, 10424752 and 1:200 Alexa-Flour-568, Invitrogen, 10348072) for $1 \mathrm{~h}$ at RT. Cells

677 were incubated with DAPI solution (AppliChem, A4099) for 10 min and stored in PBS at 4C until 678 the analysis.

679

680 SA- $\beta$-gal was detected using senescence cells histochemical staining kit (Sigma-Aldrich, 681 CS0030) according to manufacturer's protocol. Cell colonies were imaged using INcell analyzer 6822200 high content microscopy at 20x magnification to produce 1199 images with $2048 \times 2048$ 
683 pixel resolution. Due to system constraints for object detection, each image was split into four 684 tiles of $1024 \times 1024$ pixel resolution.

686 Cell Cycle Analysis

687 Fibroblast cell lines AG08498, GM22159 and GM22222 were seeded in 6-well cell culture plates 688 and 24 hours later DNA-damage induced senescence was conducted as described above (5

689 Gy, 10 Gy or 20 Gy). After 9 days cells were harvested by trypsinization and washed twice with 690 PBS. Cells were fixed by adding dropwise ice-cold $70 \%$ ethanol while mixing the cells gently on 691 a vortex mixer. Thereafter, the cells were incubated for $30 \mathrm{~min}$ on ice and washed twice in PBS. 692 Fixed cells were incubated with RNase $\left(100 \mu \mathrm{g} / \mathrm{ml}\right.$; ThermoFisher Scientific) at $37^{\circ} \mathrm{C}$ for $30 \mathrm{~min}$. 693 Propidium iodide (20 $\mathrm{mg} / \mathrm{ml}$; Sigma-Aldrich) was added and incubated for $30 \mathrm{~min}$ at $37^{\circ} \mathrm{C}$. Cell 694 cycle status was determined by flow cytometry (CytoFlex, Beckman Coulter).

\section{Nuclei Detection}

697 A base library was prepared using control, irradiated (IR), and cells serially passaged until they 698 reached senescence (replicative senescence, RS). A deep neural network model was applied to 699 detect DAPI-stained nuclei. The samples were used to build a training set for nuclei recognition.

700 Several images were selected arbitrarily from each group for a total of $\sim 20$ samples, and all

701 nuclei in the training samples were annotated by selecting the nuclear region. U-NET, a 23-layer

702 fully convolutional network for image segmentation, was trained using the samples, learning to

703 associate the DAPI images with annotation masks indicating nuclear regions. Our

704 implementation of U-NET is largely based on the original U-NET ${ }^{47}$, but includes a dropout layer

705 after each of the convolutional and deconvolutional layers to reduce overfitting. After training for

7061000 epochs, the U-NET model was used to detect nuclei for all 4796 tiles (1199 images $x 4$

707 tiles/image), producing output images of predicted nuclei regions. The images with predicted

708 nuclei were scanned for recognition regions of area between 500 and 15,000 pixels. Each 
detected nucleus was extracted along with its surrounding context as a centered $128 \times 128$ pixel

710 region and used to assemble a base library of 95,152 nuclei. In addition, the recognition region

711 itself was cutout, providing a two-color reduction of the detected nuclei, and assembled into a

712 secondary library of nuclei masks.

713

\section{$714 \quad$ Nuclear Morphology}

715 An analysis of the nuclei was performed to assess morphological properties. The two-color

716 mask library was used, since it provided a universal representation of the detected nuclei (with

717 U-NET detector models that have good coverage of the nuclei region). Nuclear morphology was

718 assessed using several metrics, including area, perimeter, moments, convexity, and aspect

719 ratio. Convexity is the ratio of perimeter to convex hull perimeter, which provides a size-neutral

720 measure of boundary regularity. The convex hull is a polygon that connects the outer edges of

721 nuclei like an envelope.

722

723 Senescent Classification

724 After assembling a library of senescent cells, a deep neural network was trained to classify

725 DAPI-stained nuclei as senescent or non-senescent. The training set was based on several cell

726 lines GM22159, GM03349, GM05757, while additional cell lines GM22222 and AG08498 were

727 used for testing. Training samples were randomized and split into $80 \%$ for training and $20 \%$ for

728 validation. Due to experimental setup, the sample classes are unbalanced, with $75.2 \%$ control,

$72911.2 \%$ RS, and $13.6 \%$ IR. The samples were balanced during training by applying class weights

730 with inverse proportion to the class abundance (for example, senescent samples composed of

731 IR and RS were fewer in number and therefore valued 3x higher than controls). Image samples

732 were normalized for brightness/intensity by adjusting each image's mean intensity to 0 and

733 standard deviation to 1. Augmentation was also applied during training, randomly modifying

734 samples: adjusting size from $80 \%$ to $120 \%$, changing normalized brightness from $70 \%$ to $130 \%$, Page 30 of 44 
735 flipping horizontally and vertically, and rotating up to 180 degrees. For each epoch, one

736 augmentation cycle was performed. Training was done with Xception, a 48-layer model,

737 initialized with ImageNet weights but set to allow weight adjustment of all layers during training.

738 The top layer was replaced by a layer of one-hot nodes to indicate the state as controls or

739 senescent (or as a tri-state model with controls, IR, or RS to indicate the type of senescence).

740 With this minor adjustment, the model provided $37,640,234$ trainable parameters. Training was

741 done using Adam with the learning rate set to $1 \times 10^{-4}$ for 10 epochs, in which time accuracy

742 rapidly converged to a steady level. In addition, a simpler custom model was tested, with three

743 convolutional layers with ReLU activation and two dense layers with L1/L2 regularization of

$744 \quad 0.05 / 0.05$ and $30 \%$ dropout. This model required 713,296 parameters. For both network

745 designs, we trained with raw images along with several modified image sets, where the

746 background was removed, the nuclei were size normalized, and the inner details of nuclei were

747 entirely masked (Fig. 1A). All three techniques were based on the detected nuclei. To remove

748 the background, the area outside of the nuclei was set to 0 . Size was normalized by rescaling all

749 nuclei so the larger of the two dimensions was a standard size of 80 pixels. Finally, the size-

750 normalized detection region was used for the masked nuclei set.

751

752 Bayesian Neural Network

753 We used Tensorflow Probability to create a Bayesian neural network (BNN). We first converted

754 the simple custom model, replacing nodes with the comparable FlipOut version ${ }^{28}$, which

755 assumes that the kernel and bias are drawn from a normal distribution. During a forward pass,

756 kernels and biases are sampled from posterior distribution. Targets were encoded as above,

757 and the loss function used was cross entropy plus KL divergence divided by number of batches.

758 We also partially converted Xception to a BNN by replacing all dense and convolutional layers

759 to FlipOut nodes, leaving separable convolutions unconverted since a FlipOut version was not

760 available. In addition, we fully converted InceptionV3 for evaluation. Inference was done by

Page 31 of 44 
761 evaluating the model 20 times to produce a distribution of predictions, and then taking the mean

762 probability for each sample.

764 Deep Neural Network Ensemble

765 To improve accuracy and provide a more robust solution, we also worked with an ensemble of

766 deep learning models. This method utilized 10 models of Xception, each trained on the same

767 data set with different random weight initialization. To generate predictions, each model

768 instance was applied, and the results combined by taking the mean prediction. We also tried

769 bagging, also known as bootstrap aggregation. Similar to the deep ensemble, this method trains

770 different model instances with bootstrap selection of samples for $n=1-1 / e$. With each instance

771 trained on a different subset of samples, this method produces multiple models that in theory

772 can specialize to different sets of data.

773

774 Statistical Methods

775 All comparisons with between groups of samples were made using one-way ANOVA f-tests to

776 evaluate differences in the means, followed by pair-wise tests using Tukey's HSD (Honest

777 Significant Difference) to calculate p-values between groups. Linear regression methods were

778 evaluated with $\mathrm{R}$ and p-value statistics. Groups of patients were compared using the chi-

779 squared test and Fisher test to detect significant differences between frequencies. Correlation

780 was evaluated using the Pearson colocalization coefficient.

781

782 Pathology sample selection

783 The individuals were sampled from patients for whom samples of naevi on non-sun exposed

784 skin had undergone pathology without malignant findings at a major pathology department in

785 Copenhagen. The patient sample was selected to have flat distribution of age. We selected

786 patient samples from the Danish National Register of Pathology requisitioned in 2007-2017 and Page 32 of 44 
coded with one or more PatoSNOMED topology code: T02530 (Skin on penis), T76330

788 (Foreskin), T80200 (Mons pubis), T02471(Skin on nates), T02480 (Skin on abdomen), T02430

789 (Skin on breasts) and one or more procedure code: P30620 (resect), P306X0 (ectomy

790 preparation), P30611 (excision biopsy) and one or more morphology code: M87400 (junction

791 naevus), M87500 (dermal naevus), M87600 (compound naevus).

792

793 Senescence and Human Morbidity

794 We collected ICD-10 diagnosis codes from the Danish National Patient Register in the period

$795 \quad 1977-2018$ of each of the patients in this study. We further grouped diagnoses into each of 21

796 ICD-10 chapters. We calculated the linear regression residuals of the relationship between age

797 at pathology examination and the predicted senescent cell load (IR, RS metrics) for each of the

798 patients. We then constructed contingency tables counting the number of patients with and

799 without a specific diagnosis and with a predicted senescent cell load above or below the age-

800 dependent average. We used Pearson's chi-squared test and Fisher's exact test to determine

801 whether patients with a predicted senescent cell load above or below the age-dependent

802 average were associated with a higher or lower incidence of specific diagnosis codes (or

803 diagnosis within a specific ICD-10 chapter.)

804

\section{Animals}

806 Male C57BL/6J mice were acquired from Janvier Labs (Le Genest Saint Isle, France). Animals

807 arrived at 5-8 weeks of age and were housed in a controlled environment (12 h light/dark cycle,

$80821 \pm 2{ }^{\circ} \mathrm{C}$, humidity $50 \pm 10 \%$ ). Stratification and randomization into individual diet groups were

809 based on baseline body weight. Mice had ad libitum access to tap water and chow (2018 Teklad

810 Rodent Diet, Envigo, Madison, WI, United States; Altromin 1324, Brogaarden, Hoersholm,

811 Denmark). The study was approved by The Institutional Animal Care and Use Committee at

812 Medlmmune (Gaithersburg, MD, United States) and The Danish Animal Experiments

Page 33 of 44 
813 Inspectorate (license: 2017-15-0201-01378) and performed in accordance with internationally

814 accepted principles for the use of laboratory animals.

815

$816 \quad$ Liver histology

817 Terminal liver samples were dissected from the left lateral lobe immediately after sacrificing the 818 animal and subsequently fixed overnight in 4\% paraformaldehyde. The liver tissue was then

819 paraffin-embedded and sectioned at a thickness of $3 \mu \mathrm{m}$. Sections were stained with

820 hematoxylin-eosin (HE, Dako, Glostrup, Denmark). Slides were scanned by ScanScope AT

821 System (Aperio, Vista, CA, United States).

822

823

824 


\section{Acknowledgements}

826 This research was supported by the Novo Nordisk Foundation Challenge Programme

827 (\#NNF17OC0027812), the Nordea Foundation (\#02-2017-1749), the Neye Foundation, the

828 Lundbeck Foundation (\#R324-2019-1492), the Ministry of Higher Education and Science

829 (\#0238-00003B), VitaDAO and Insilico Medicine. M.L.I. and M.G. were supported by the NIA

830 IRP, NIH.

831

\section{Author Contributions}

833 I.H. wrote the article, developed and trained deep learning models, and analyzed data. G.V.M.

834 performed experiments on the base data set, astrocytes, neurons, and premature aging disease.

835 M.B.E. analyzed clinical data. D.B. performed flow experiments. J.S.M. developed Bayesian

836 networks and advised the project. M.H.N. and D.O. performed animal experiments. M.L.I. and M.G.

837 shared images of human dermal tissue from their study and edited the project. L.M. managed clinical

838 images and medical records. E.V. advised and edited the project. R.W. advised and edited the

839 project. M.S.K. conceived the idea, supervised the project and edited the manuscript.

840

\section{Declaration of Interests}

842 The authors declare no competing interests. 


\section{References}

1. Kirkland, J. L. \& Tchkonia, T. Cellular Senescence: A Translational Perspective.

$847 \quad$ EBioMedicine 21, 21-28 (2017).

848 2. Zglinicki, T. von, Saretzki, G., Ladhoff, J., Fagagna, F. d'Adda di \& Jackson, S. P. Human

849 cell senescence as a DNA damage response. Mech. Ageing Dev. 126, 111-117 (2005).

850 3. Covarrubias, A. J. et al. Senescent cells promote tissue NAD+ decline during ageing via the 851 activation of CD38+ macrophages. Nat. Metab. 2, 1265-1283 (2020).

852 4. Childs, B. G. et al. Senescent cells: an emerging target for diseases of ageing. Nat. Rev. 853 Drug Discov. 16, 718-735 (2017).

854 5. Schafer, M. J. et al. The senescence-associated secretome as an indicator of age and $855 \quad$ medical risk. JCl Insight 5, e133668 (2020).

856 6. Young, A. R. J., Narita, M. \& Narita, M. Cell Senescence as Both a Dynamic and a Static 857 Phenotype. in Cell Senescence (eds. Galluzzi, L., Vitale, I., Kepp, O. \& Kroemer, G.) vol. $858 \quad 9651-13$ (Humana Press, 2013).

859 7. Basisty, N. et al. A proteomic atlas of senescence-associated secretomes for aging 860 biomarker development. PLOS Biol. 18, e3000599 (2020).

861 8. Matjusaitis, M., Chin, G., Sarnoski, E. A. \& Stolzing, A. Biomarkers to identify and isolate 862 senescent cells. Ageing Res. Rev. 29, 1-12 (2016).

863 9. Ogrodnik, M. Cellular aging beyond cellular senescence: Markers of senescence prior to cell $864 \quad$ cycle arrest in vitro and in vivo. Aging Cell (2021) doi:10.1111/acel.13338.

865 10. Lee, S. \& Schmitt, C. A. The dynamic nature of senescence in cancer. Nat. Cell Biol. 21, 8669 94-101 (2019).

867 11. Campisi, J. Cellular senescence: putting the paradoxes in perspective. Curr. Opin. Genet. 868 Dev. 21, 107-112 (2011). 
12. Gorgoulis, V. et al. Cellular Senescence: Defining a Path Forward. Cell 179, 813-827

870 (2019).

871 13. Mitsui, Y. \& Schneider, E. L. Increased nuclear sizes in senescent human diploid fibroblast $872 \quad$ cultures. Exp. Cell Res. 100, 147-152 (1976).

873 14. Chen, J.-H. \& Ozanne, S. E. Deep senescent human fibroblasts show diminished DNA 874 damage foci but retain checkpoint capacity to oxidative stress. FEBS Lett. 580, 6669-6673 875 (2006).

876 15. Kusumoto, D. et al. Anti-senescent drug screening by deep learning-based morphology 877 senescence scoring. Nat. Commun. 12, 257 (2021).

878 16. Campisi, J. CANCER: Suppressing Cancer: The Importance of Being Senescent. Science $879 \quad 309,886-887(2005)$.

880 17. Collado, M. \& Serrano, M. Senescence in tumours: evidence from mice and humans. Nat. $881 \quad$ Rev. Cancer 10, 51-57 (2010).

882 18. Collado, M., Blasco, M. A. \& Serrano, M. Cellular Senescence in Cancer and Aging. Cell $883 \quad 130,223-233(2007)$.

884 19. Zhao, H. \& Darzynkiewicz, Z. Biomarkers of Cell Senescence Assessed by Imaging 885 Cytometry. in Cell Senescence (eds. Galluzzi, L., Vitale, I., Kepp, O. \& Kroemer, G.) vol. 886965 83-92 (Humana Press, 2013).

887 20. Goldman, R. D. et al. Accumulation of mutant lamin A causes progressive changes in 888 nuclear architecture in Hutchinson-Gilford progeria syndrome. Proc. Natl. Acad. Sci. 101, 889 8963-8968 (2004).

890 21. Martins, F., Sousa, J., Pereira, C. D., Cruz e Silva, O. A. B. \& Rebelo, S. Nuclear envelope 891 dysfunction and its contribution to the aging process. Aging Cell 19, (2020).

892 22. Baus, F. Permanent cell cycle exit in G2 phase after DNA damage in normal human 893 fibroblasts. EMBO J. 22, 3992-4002 (2003).

894 23. Gire, V. \& Dulić, V. Senescence from G2 arrest, revisited. Cell Cycle 14, 297-304 (2015). 
895

896

897

898

899

900

901

902

903

904

905

906

907

908

909

910

911

912

913

914

915

916

917

918

919

920

24. Kassani, S. H., Kassani, P. H., Wesolowski, M. J., Schneider, K. A. \& Deters, R. Breast Cancer Diagnosis with Transfer Learning and Global Pooling. in 2019 International Conference on Information and Communication Technology Convergence (ICTC) 519-524 (IEEE, 2019). doi:10.1109/ICTC46691.2019.8939878.

25. Tomita, H. et al. Deep Learning for the Preoperative Diagnosis of Metastatic Cervical Lymph Nodes on Contrast-Enhanced Computed ToMography in Patients with Oral Squamous Cell Carcinoma. Cancers 13, 600 (2021).

26. Debacq-Chainiaux, F., Erusalimsky, J. D., Campisi, J. \& Toussaint, O. Protocols to detect senescence-associated beta-galactosidase (SA-ßgal) activity, a biomarker of senescent cells in culture and in vivo. Nat. Protoc. 4, 1798-1806 (2009).

27. Gal, Y. \& Ghahramani, Z. Dropout as a Bayesian Approximation: Representing Model Uncertainty in Deep Learning. ArXiv150602142 Cs Stat (2016).

28. Wen, Y., Vicol, P., Ba, J., Tran, D. \& Grosse, R. Flipout: Efficient Pseudo-Independent Weight Perturbations on Mini-Batches. ArXiv180304386 Cs Stat (2018).

29. Fort, S., Hu, H. \& Lakshminarayanan, B. Deep Ensembles: A Loss Landscape Perspective. ArXiv191202757 Cs Stat (2020).

30. Hewitt, G. et al. Telomeres are favoured targets of a persistent DNA damage response in ageing and stress-induced senescence. Nat. Commun. 3, 708 (2012).

31. Hernandez-Segura, A., Nehme, J. \& Demaria, M. Hallmarks of Cellular Senescence. Trends Cell Biol. 28, 436-453 (2018).

32. Petr, M. A., Tulika, T., Carmona-Marin, L. M. \& Scheibye-Knudsen, M. Protecting the Aging Genome. Trends Cell Biol. 30, 117-132 (2020).

33. Keijzers, G., Bakula, D. \& Scheibye-Knudsen, M. Monogenic Diseases of DNA Repair. N.

Engl. J. Med. 377, 1868-1876 (2017).

34. Moreno-Blas, D. et al. Cortical neurons develop a senescence-like phenotype promoted by dysfunctional autophagy. Aging 11, 6175-6198 (2019).

Page 38 of 44 
921 35. Wang, C. et al. DNA damage response and cellular senescence in tissues of aging mice:

922 Senescent cells in aging mice. Aging Cell 8, 311-323 (2009).

923 36. Burton, D. G. A. \& Krizhanovsky, V. Physiological and pathological consequences of cellular 924 senescence. Cell. Mol. Life Sci. 71, 4373-4386 (2014).

925 37. He, S. \& Sharpless, N. E. Senescence in Health and Disease. Cell 169, 1000-1011 (2017).

926 38. Nelson, D. M., McBryan, T., Jeyapalan, J. C., Sedivy, J. M. \& Adams, P. D. A comparison of 927 oncogene-induced senescence and replicative senescence: implications for tumor $928 \quad$ suppression and aging. AGE 36, 9637 (2014).

929 39. Idda, M. L. et al. Survey of senescent cell markers with age in human tissues. Aging 12, $930 \quad 4052-4066(2020)$.

931 40. Liggett, W. H. \& Sidransky, D. Role of the p16 tumor suppressor gene in cancer. J. Clin.

932 Oncol. 16, 1197-1206 (1998).

933 41. Campisi, J., Andersen, J. K., Kapahi, P. \& Melov, S. Cellular senescence: A link between 934 cancer and age-related degenerative disease? Semin. Cancer Biol. S1044579X11000502 935 (2011) doi:10.1016/j.semcancer.2011.09.001.

936 42. Burd, C. E. et al. Monitoring Tumorigenesis and Senescence In Vivo with a p16INK4a$937 \quad$ Luciferase Model. Cell 152, 340-351 (2013).

938 43. Wang, B., Kohli, J. \& Demaria, M. Senescent Cells in Cancer Therapy: Friends or Foes?

939 Trends Cancer 6, 838-857 (2020).

940 44. Pathak, R. U., Soujanya, M. \& Mishra, R. K. Deterioration of nuclear morphology and 941 architecture: A hallmark of senescence and aging. Ageing Res. Rev. 67, 101264 (2021).

942 45. Filippi-Chiela, E. C. et al. Nuclear Morphometric Analysis (NMA): Screening of Senescence, $943 \quad$ Apoptosis and Nuclear Irregularities. PLoS ONE 7, e42522 (2012).

944 46. Neri, F., Basisty, N., Desprez, P.-Y., Campisi, J. \& Schilling, B. Quantitative Proteomic 945 Analysis of the Senescence-Associated Secretory Phenotype by Data-Independent $946 \quad$ Acquisition. Curr. Protoc. 1, e32 (2021).

Page 39 of 44 
947 47. Ronneberger, O., Fischer, P. \& Brox, T. U-Net: Convolutional Networks for Biomedical 948 Image Segmentation. in Medical Image Computing and Computer-Assisted Intervention 949 MICCAI 2015 (eds. Navab, N., Hornegger, J., Wells, W. M. \& Frangi, A. F.) vol. $9351234-$ 950241 (Springer International Publishing, 2015).

951

952 


\section{Figure Legends}

955 Figure 1 Nuclear morphology is an accurate senescence predictor in cultured cells. a

956 Analysis workflow. b Sample nuclei for controls, replicative senescence (RS) and ionizing

957 radiation (IR) induced senescent cells. c Area of identified nuclei $(n=6,976-68,971$, mean $\pm 95 \%$

$958 \mathrm{Cl})$. d Convexity of identified nuclei $(n=6,976-68,971$, mean $\pm 95 \% \mathrm{Cl})$. e Aspect ratio of

959 identified nuclei $(n=6,976-68,971$, mean $\pm 95 \% \mathrm{Cl})$. f Scatter plot of individual nuclei, with

960 overall distributions for each at the top and right margins. $\mathbf{g}$ Cell cycle analysis after exposure to

961 several doses of IR; mn: multinucleated cells. h Accuracy of a deep neural network (DNN)

962 predictor on validation data. i Receiver operating characteristics (ROC) curve of the DNN. j

963 Percent of nuclei in each state classified as senescent for independent cell lines. k Distribution

964 of prediction probabilities for several doses of IR for three fibroblast cell lines $(n=38,284-$

$965106,132)$. I Distribution of p21 intensities for several doses of IR for three fibroblast cell lines 966 (n=38,284-106,132). $\mathbf{m}$ Distribution of PCNA intensities for several doses of IR for three 967 fibroblast cell lines ( $\mathrm{n}=38,284-106,132)$. $\mathbf{n}$ Correlation between predicted senescence and 968 nearby SA- $\beta$-gal regions, showing all and $90 \%$ confidence predictions only for RS and IR 969 groups. o Correlation between predicted senescence and multiple markers, showing all, filtered

970 for markers with strong signals, and filtered with $90 \%$ confidence predictions only. p Accuracy of

971 DNNs trained and predicting after different normalization methods. q Correlation between

972 morphological metrics and predicted senescence by class, BG: background.

973

974 Figure 2 Predictions from deep ensembles increases accuracy. a Heatmap of variation in

975 predictions by members of ensemble (500 sample nuclei as rows, ensemble members as

976 columns). Blue is young/control and white is senescent. b Heatmap of per-class accuracy for

977 control and senescent by ensemble model. c Accuracy of deep ensemble. $\mathbf{d}$ ROC curve for the 
978 deep ensemble. e Accuracy of single model, Bayesian neural networks, deep ensemble, and

979 bagging. f Accuracy of deep ensemble with normalized samples. g ROC curve for the deep

980 ensemble with normalized samples. h Accuracy of three-state senescence ensemble model. i

981 ROC curve for the three-state senescence ensemble model. $\mathbf{j}$ Accuracy of RS-only model. $\mathbf{k}$

982 Accuracy of IR-only model.

983

984 Figure 3 Senescence can be predicted across tissues and species. a Number of $\gamma \mathrm{H} 2 \mathrm{AX}$

985 foci by type of senescence $(n=1,831-15,560$, mean $\pm 95 \%$ Cl). b Number of 53BP1 foci by type

986 of senescence $(n=1,831-15,560$, mean $\pm 95 \% \mathrm{Cl})$. c Correlation between foci count and

987 predicted senescence. d Representative immunohistochemistry micrographs of premature

988 aging nuclei with DNA damage foci staining of $\mathrm{\gamma H} 2 \mathrm{AX}$ and 53BP1, HGPS: Hutchinson-Gilford

989 Progeria Syndrome, AT: ataxia telangiectasia, CS: Cockayne Syndrome. e Nuclear area for

990 premature aging diseases $(n=4,340-15074$, mean $\pm 95 \% \mathrm{Cl})$, HGPS: Hutchinson-Gilford

991 Progeria Syndrome, AT: ataxia telangiectasia, CS: Cockayne Syndrome. f Number of $\mathrm{\gamma H} 2 \mathrm{AX}$

992 foci for premature aging diseases $(n=5,162-17,584$, mean $\pm 95 \%$ Cl). $\mathbf{g}$ Number of 53BP1 foci

993 by premature aging diseases $(n=5,162-17,584$, mean $\pm 95 \% \mathrm{Cl})$. $\mathrm{h}$ Predicted probability of

994 senescence for premature aging disease $(n=5,162-17,584$, mean $\pm 95 \% \mathrm{Cl})$. i Nuclei with

995 nearby SA- $\beta$-gal regions. j Correlation between predicted senescence and nearby SA- $\beta$-gal and

996 also number of samples, as confidence filtering is applied across a range of thresholds. $\mathbf{k}$ DAPI

997 intensities for premature aging diseases and controls $(n=4,340-15,074$, mean $\pm 95 \% \mathrm{Cl})$. I

998 Representative immunohistochemistry micrographs of senescent murine astrocytes with DNA

999 damage foci staining of $\mathrm{\gamma H} 2 \mathrm{AX}$ and 53BP1. $\mathrm{m}$ Nuclear area of murine astrocytes $(\mathrm{n}=4,888-$

$100013,549$, mean $\pm 95 \% \mathrm{Cl})$. $\mathbf{n}$ Nuclear area of murine neurons $(\mathrm{n}=33,303-62,847$, mean $\pm 95 \%$

$1001 \mathrm{Cl})$. o Number of $\mathrm{\gamma H} 2 \mathrm{AX}$ foci for murine astrocytes ( $\mathrm{n}=4,918-13,661$, mean $\pm 95 \% \mathrm{Cl})$. $\mathbf{p}$

1002 Number of 53BP1 foci for murine astrocytes ( $n=4,918-13,661$, mean $\pm 95 \% \mathrm{Cl})$. q Number of

$1003 \mathrm{\gamma H} 2 \mathrm{AX}$ foci for murine neurons $(n=33,303-62,847$, mean $\pm 95 \% \mathrm{Cl})$. $\mathbf{r}$ Number of $53 \mathrm{BP} 1$ foci for 
1004 murine neurons $(n=33,303-62,847$, mean $\pm 95 \% \mathrm{Cl})$. s Predicted senescence for murine 1005 astrocytes $(n=4,918-13,661$, mean $\pm 95 \% \mathrm{Cl})$. t Predicted senescence for murine neurons $1006(n=33,303-62,847$, mean $\pm 95 \% \mathrm{Cl})$.

1007

1008 Figure 4 Senescence can be predicted across tissues and species. a Analysis workflow. b 1009 Mean nuclear area per mouse by age $(n=5)$. c Mean nuclear convexity per mouse by age $(n=5)$. 1010 d Mean nuclear aspect ratio per mouse by age $(n=5)$. e Prediction probability for RS senescent $1011(n=5)$. f Prediction probability for IR senescent $(n=5)$. g Predicted percent that are RS senescent $1012(n=5)$. $h$ Predicted percent that are IR senescent $(n=5)$. i Mean probability of predicted RS 1013 senescence for each individual by p21 state. $\mathbf{j}$ Mean probability of predicted IR senescence for 1014 ech individual by p21 state. k PCNA intensity after senescence induction for three fibroblast cell 1015 lines $(n=30,957-119,669$, mean $\pm 95 \% \mathrm{Cl})$. I Area after senescence induction for three fibroblast 1016 cell lines $(n=30,957-119,669$, mean $\pm 95 \% \mathrm{CI})$. m Convexity after senescence induction for 1017 three fibroblast cell lines $(n=30,957-119,669$, mean $\pm 95 \% \mathrm{Cl})$. $\mathbf{n}$ Aspect after senescence 1018 induction for three fibroblast cell lines ( $n=30,957-119,669$, mean $\pm 95 \% \mathrm{Cl})$. o DAPI intensity 1019 after senescence induction for three fibroblast cell lines ( $n=30,957-119,669$, mean $\pm 95 \% \mathrm{Cl}) . \mathbf{p}$ 1020 Predicted probability of senescence after senescence induction, using the RS model $(n=30,957$ 1021119,669 , mean $\pm 95 \%$ Cl). q Accuracy of doxorubicin-only model. $\mathbf{r}$ Accuracy of ATV/r-only 1022 model. s Accuracy of Antimycin-A-only model. t Accuracy of unified model.

\section{Figure 5 Nuclear morphology predict senescence and multiple diseases in humans. a}

1025 Predicted probability of senescence for p21- and p21+ nuclei in human dermis, across a range 1026 of thresholds. b Analysis workflow. c Mean nuclear area per patient by age $(n=148)$. d Mean 1027 nuclear convexity per patient by age $(n=148)$. e Mean nuclear aspect ratio per patient by age $1028(n=148) . \mathbf{f}$ Predicted percent that are RS senescent $(n=169)$. $g$ Predicted percent that are IR 1029 senescent $(n=169) . \mathbf{h}$ Volcano plot of ICD-10 chapters based on IR senescence residuals and Page 43 of 44 
1030 chi-squared p-values. i Volcano plot of ICD-10 chapters based on RS senescence residuals and

1031 chi-squared p-values. j Volcano plot of ICD-10 chapters based on doxorubicin senescence

1032 residuals and chi-squared p-values. $\mathbf{k}$ Volcano plot of ICD-10 chapters based on ATV/r

1033 senescence residuals and chi-squared p-values. I Volcano plot of ICD-10 chapters based on

1034 antimycin-A senescence residuals and chi-squared p-values. $\mathbf{m}$ Volcano plot of ICD-10 chapters

1035 based on unified senescence residuals and chi-squared $p$-values. $\mathbf{n}$ Table summarizing disease

1036 conditions, percent of individuals with the condition for positive and negative residual groups, p-

1037 value from chi-squared test or Fisher's exact test, and relative risk ratio with $95 \% \mathrm{Cl}$ when

1038 significant.

1039 
Supplementary Figures 
A

IR (10 Gy)

Imaging

Seeding

\begin{tabular}{|l|l|}
\hline 1 day & 9 days
\end{tabular}

B

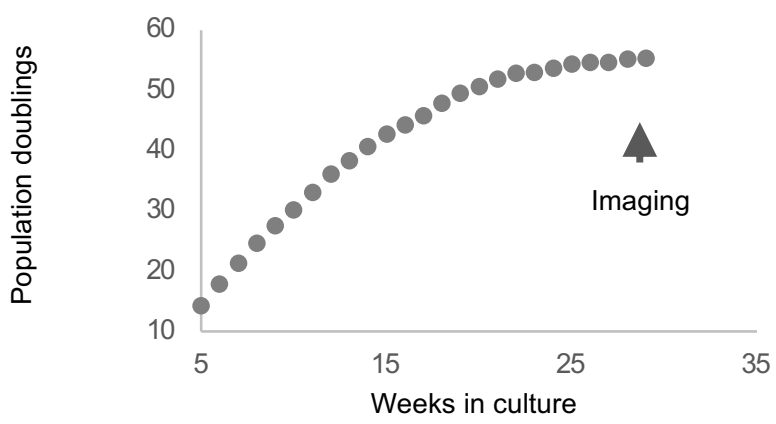

E
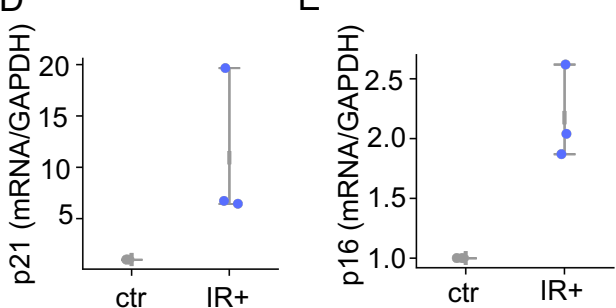

C

Control

IR
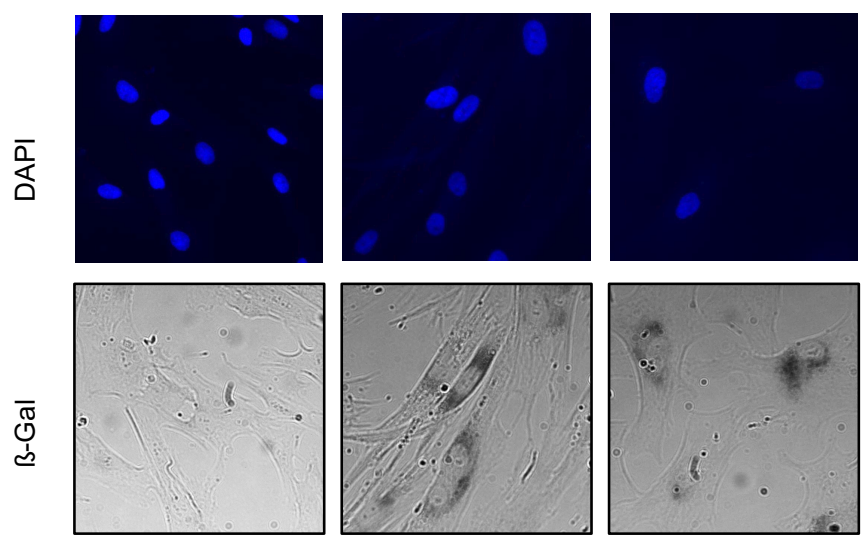

G
$\mathrm{H}$

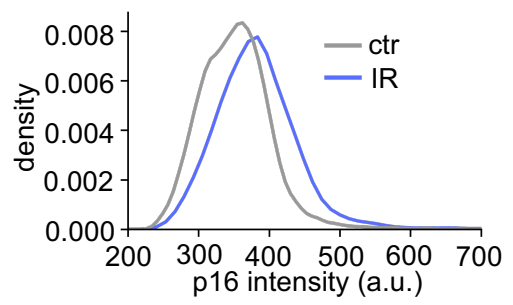

J

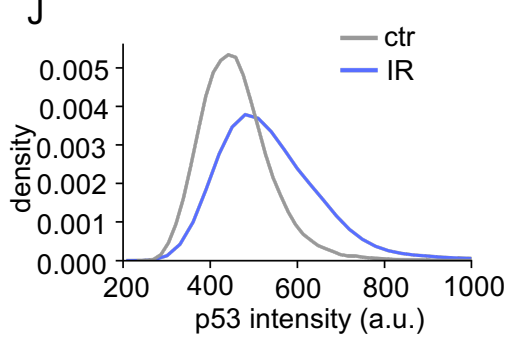

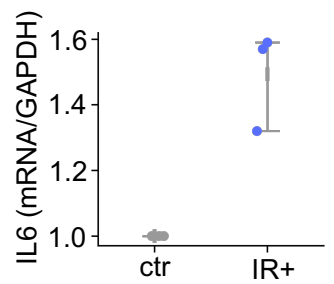

p16

I

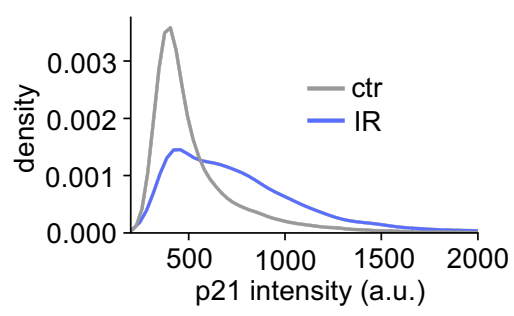

p53

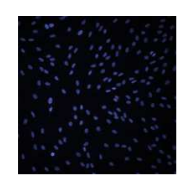

Control
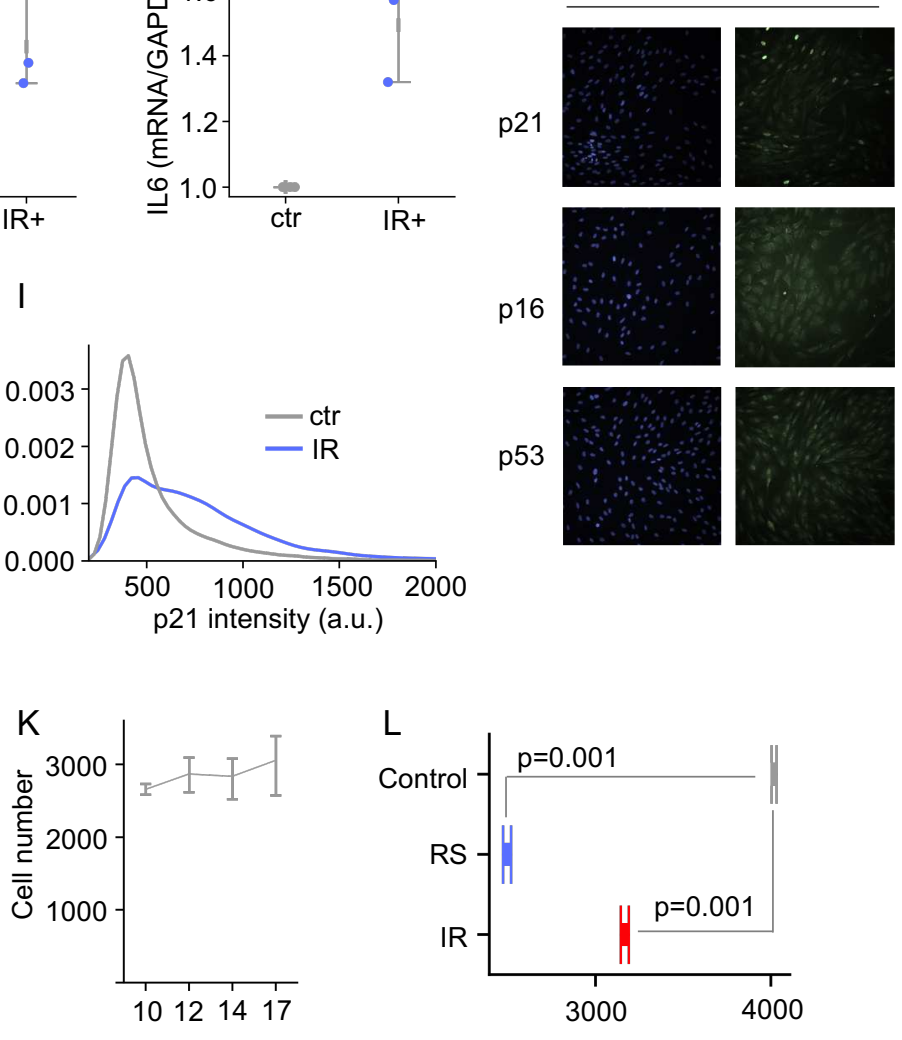

Days after IR
L

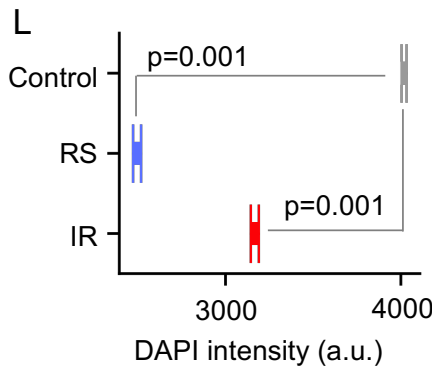

Figure S1 Cell culture models of senescence. a Schematic of lonizing radiation (IR)-induced senescence. $\mathbf{b}$ Representative growth curve of cells undergoing replicative senescence (RS). $\mathbf{c}$ Representative micrographs of SA- $\beta$-gal activity and DAPI in control, IR and RS cells. $\mathbf{d}$ Relative p21 mRNA expression levels by qPCR $(n=3, \pm$ SEM). e Relative $p 16$ mRNA expression by $q P C R(n=3, \pm$ SEM).. $f$ Relative IL6 mRNA expression by qPCR $(n=3, \pm$ SEM). $\mathbf{g}$ Representative immunohistochemistry micrographs of nuclei with $p 21, p 16$, and $p 53$ staining in control and IR cells. $\mathbf{h}$ Distribution of p16 intensities for IR and control for three fibroblast cell lines $(n=9,196-27,716)$. i Distribution of p21 intensities for IR and control for three fibroblast cell lines ( $n=13,678-32,730)$. j Distribution of p53 intensities for IR and control for three fibroblast cell lines $(n=12,844-31,100)$. $\mathbf{k}$ Cell count following irradiation. I DAPI intensities for IR, RS, and control for three fibroblast cell lines ( $n=2,641-21,954)$. 


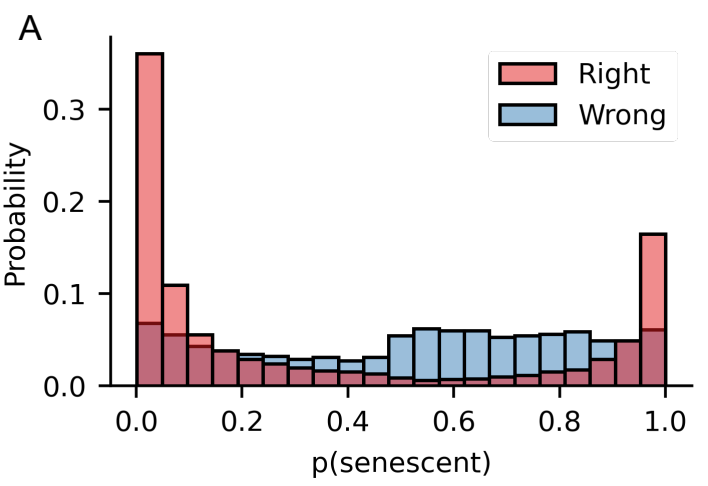

C Single Neural Network

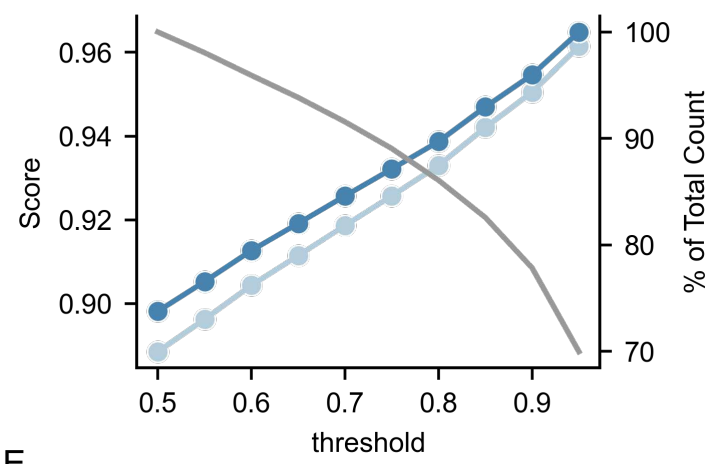

E

Ensemble

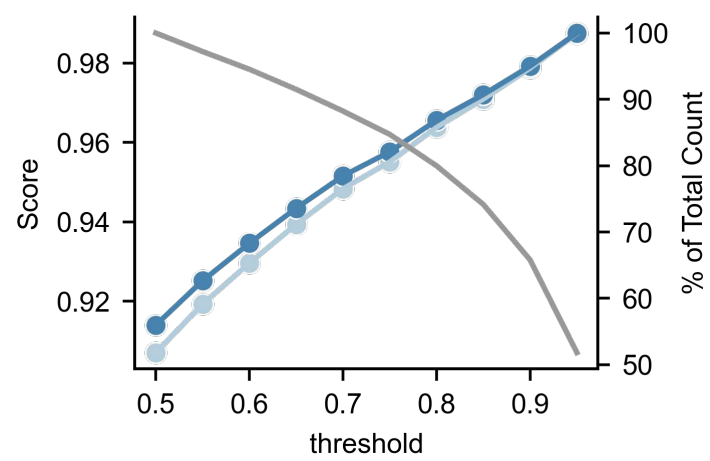

G

RS Only

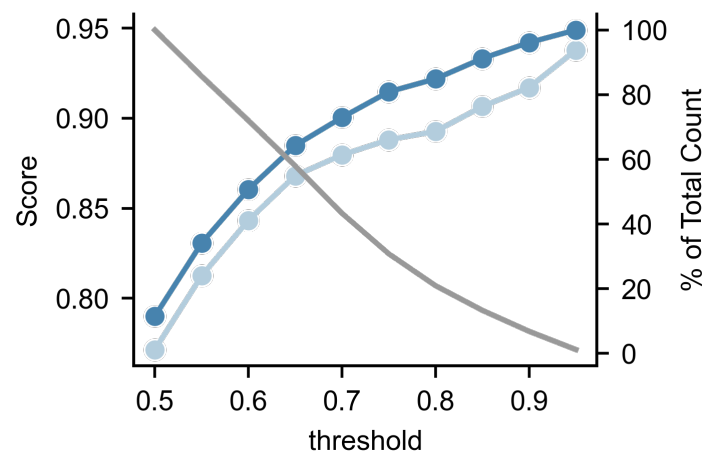

B

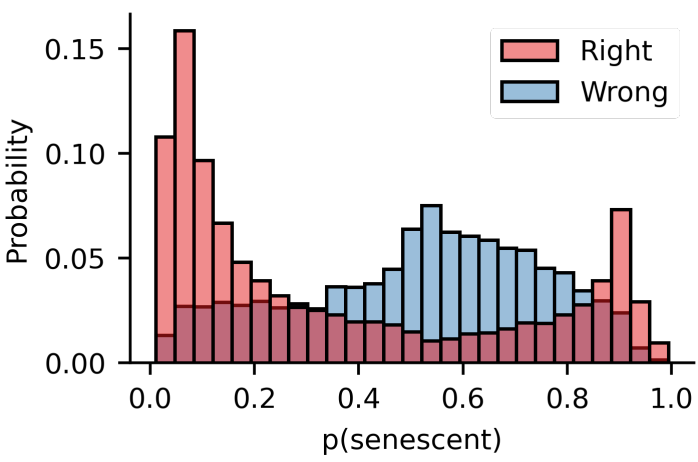

D

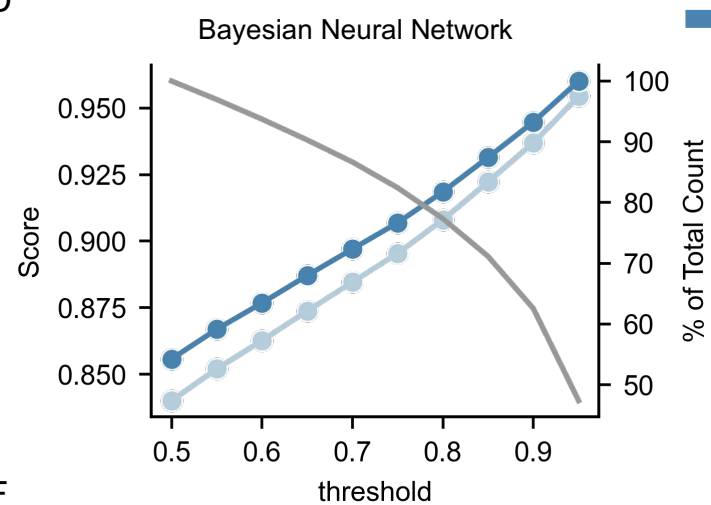

F

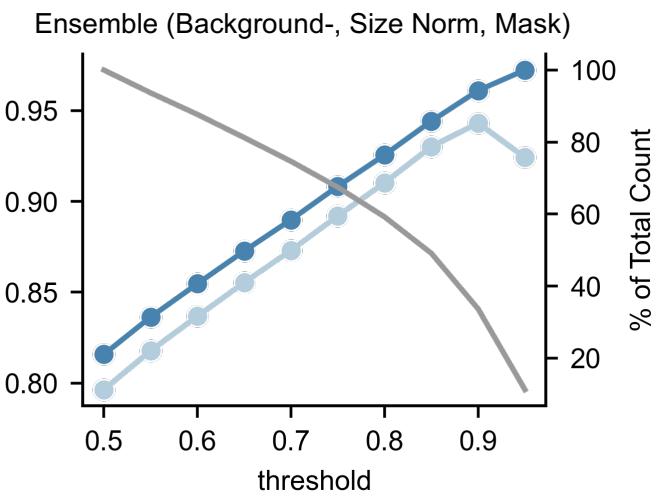

$\mathrm{H}$

IR Only

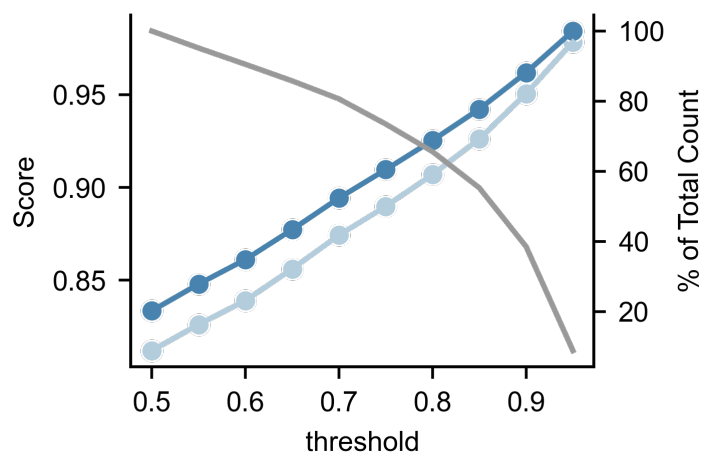

Figure S3 Improving performance by adjusting thresholds. a Histogram of predicted probabilities for Bayesian neural network. b Histogram of predicted probabilities for deep ensemble. c Accuracy and percent of samples evaluated with different classifier thresholds for single model. $\mathbf{d}$ Accuracy and percent of samples evaluated with different classifier thresholds for Xception-based Bayesian neural network. e Accuracy and percent of samples evaluated with different classifier thresholds for deep ensemble. $\mathbf{f}$ Accuracy and percent of samples evaluated with different classifier thresholds for deep ensemble with normalized samples. $g$ Accuracy and percent of samples evaluated with different classifier thresholds for ensemble of RS-only for normalized samples. $\mathbf{h}$ Accuracy and percent of samples evaluated with different classifier thresholds for ensemble of IR-only for normalized samples. 


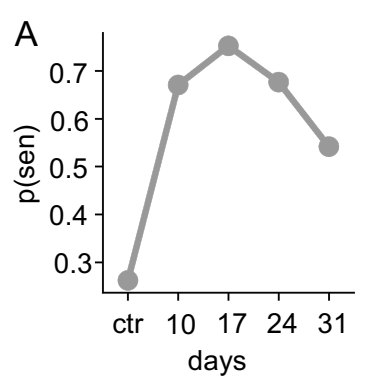

B

C

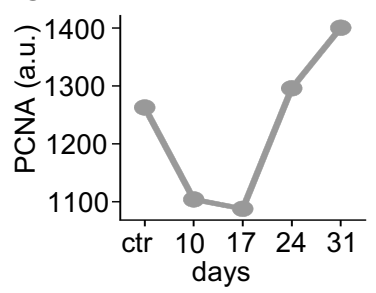

F
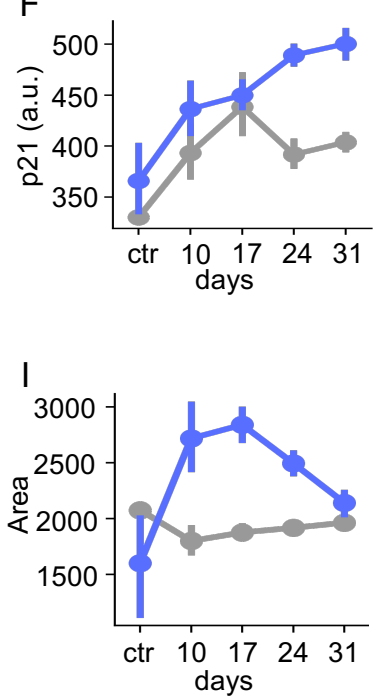

L

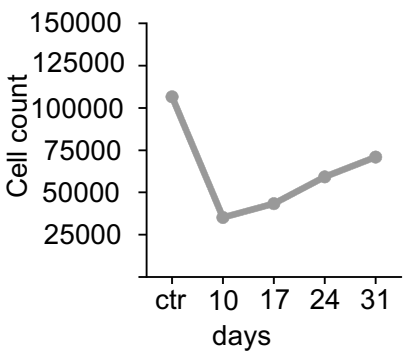

control
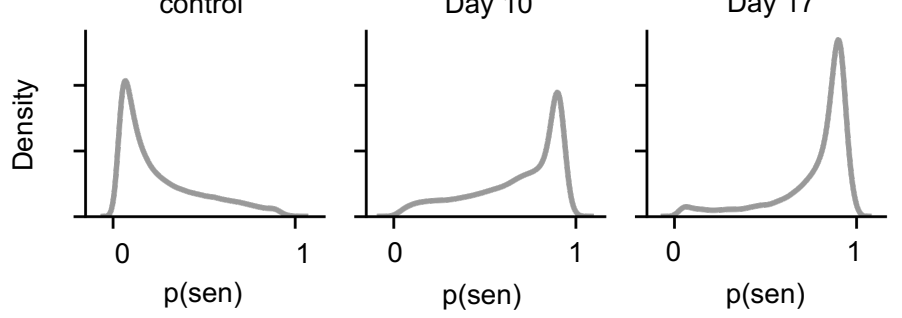

E
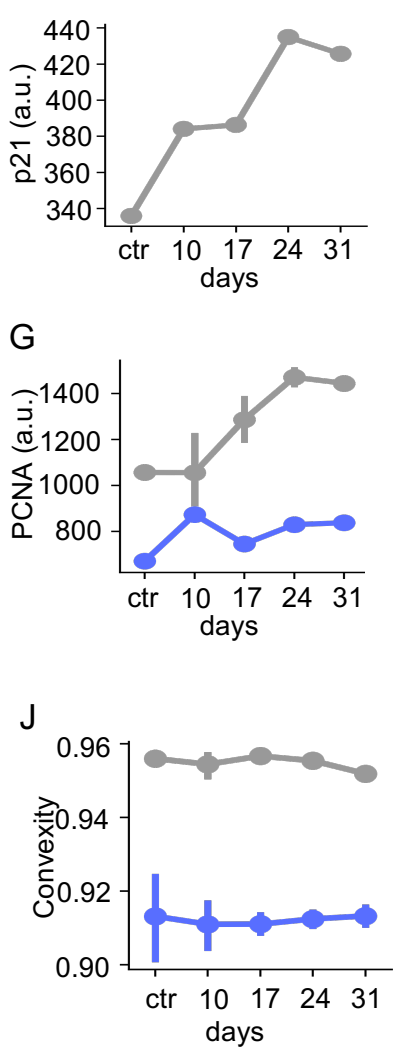

D

G

$\mathrm{H}$

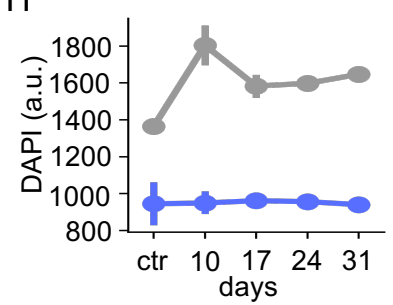

K

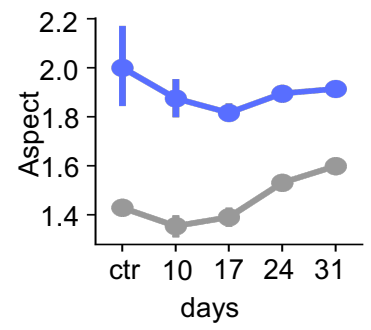

Day 24

Day 31
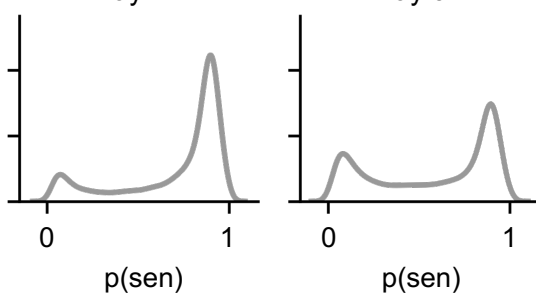

- $p($ sen $)<0.1$

- $p($ sen $)>0.9$

Figure S4 Development of Senescent Phenotype. a Predicted probability of senescence for several time points of IR-induced senescent cells for three fibroblast cell lines $(n=35,191-106,549)$. b Distribution of predicted probability split by time points. c Mean PCNA intensity per nucleus for several time points for three fibroblast cell lines $(n=35,191-106,549)$. d Mean p21 intensity per nucleus for several time points for three fibroblast cell lines $(n=35,191-106,549)$. e Mean DAPI intensity per nucleus for several time points for three fibroblast cell lines ( $n=35,191-106,549)$. $\mathbf{f} p 21$ for several time points, split by predicted senescence state. g PCNA for several time points, split by predicted senescence state. $\mathbf{h}$ DAPI for several time points, split by predicted senescence state. i Area for several time points, split by predicted senescence state. j Convexity for several time points, split by predicted senescence state. $\mathbf{k}$ Aspect for several time points, split by predicted senescence state. I Cell counts for several time points. 


\section{A}

Control
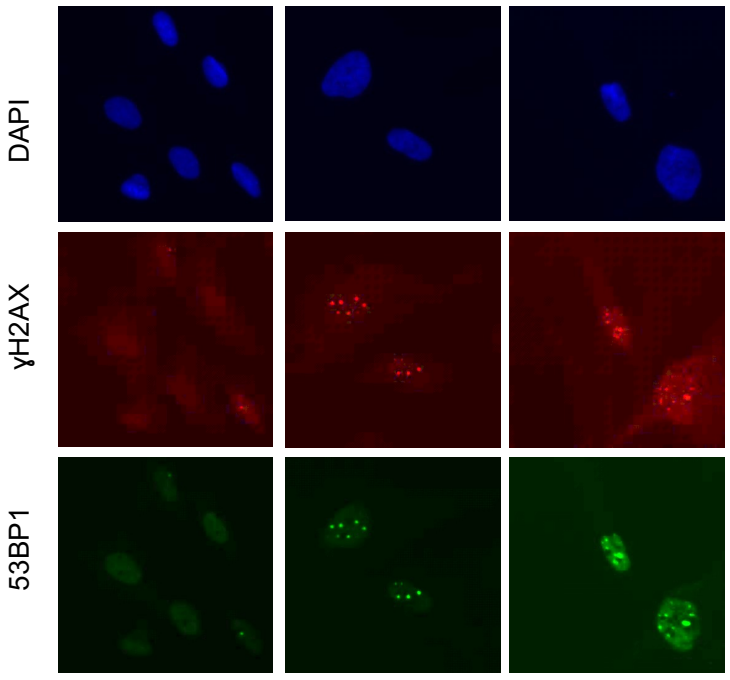

C
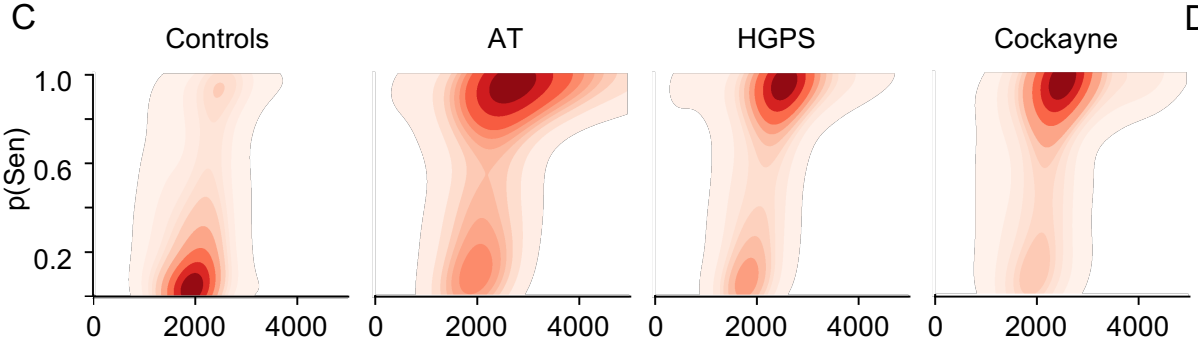

D

Astrocytes control
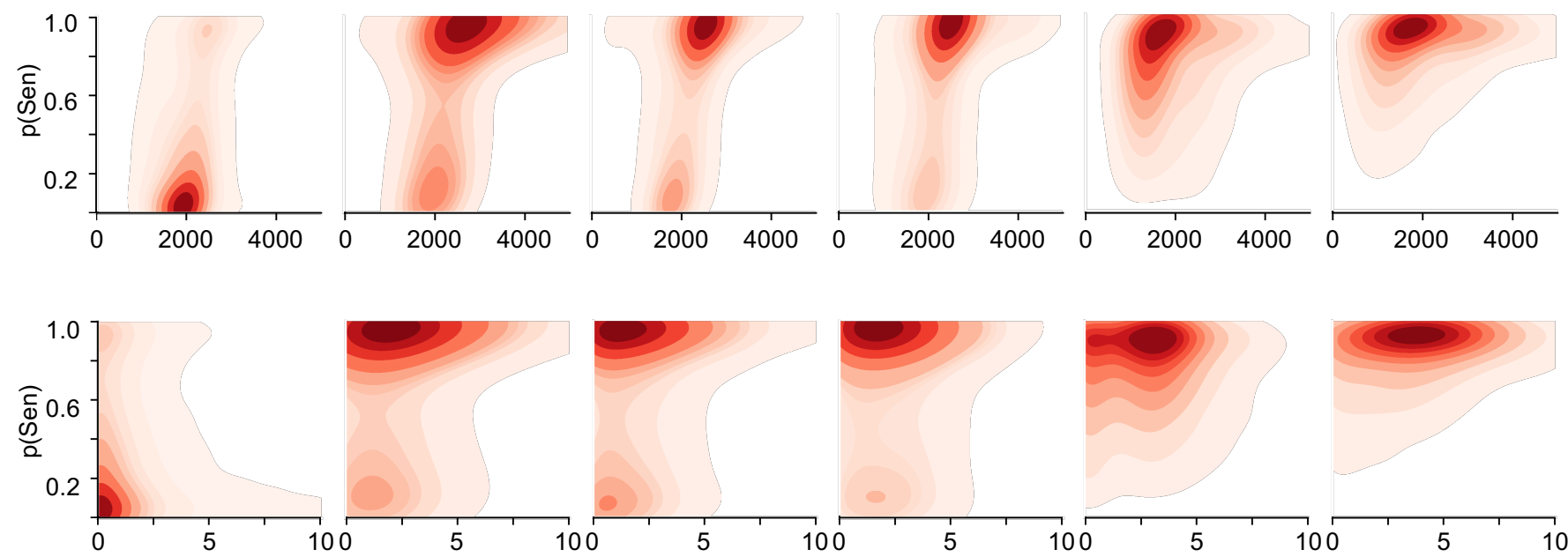

$\frac{x}{\frac{1}{x}}$

$\frac{1}{0}$
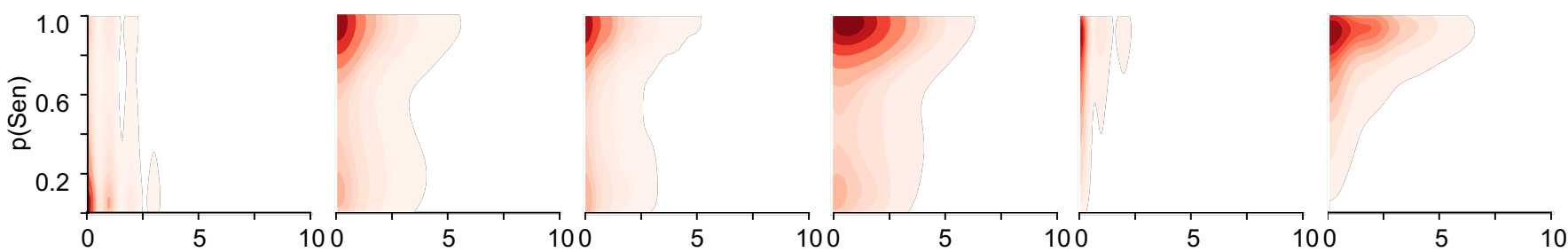

$\frac{5}{n}$

Figure S5 DNA Damage Foci and Morphological Characteristics of Predicted Senescence. a Representative immunohistochemistry micrographs of nuclei with DNA damage foci staining of gH2AX and 53BP1. b 2D histogram (density heatmap) of predicted senescence and foci count per senescence type. c 2D histogram (density heatmap) of predicted senescence and foci count for premature aging diseases. $\mathbf{d} 2 \mathrm{D}$ histogram (density heatmap) of predicted senescence and foci count for murine astrocytes. 

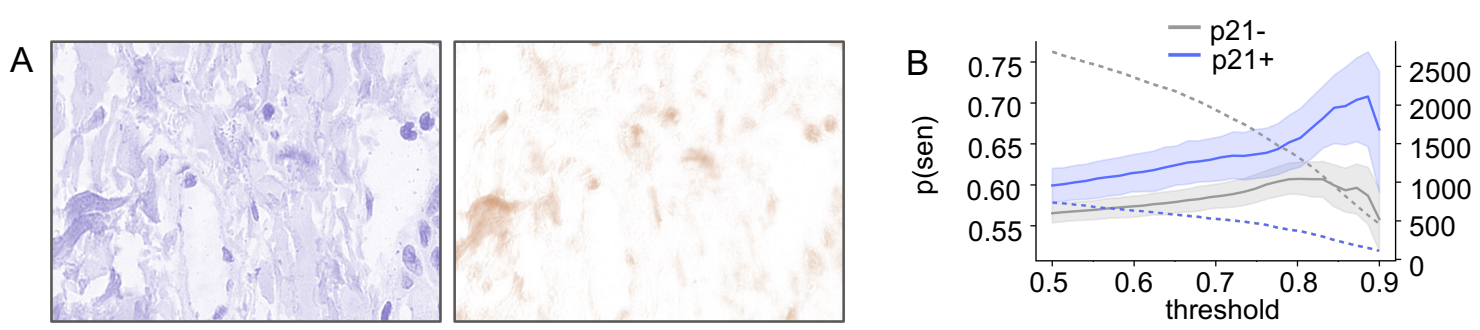
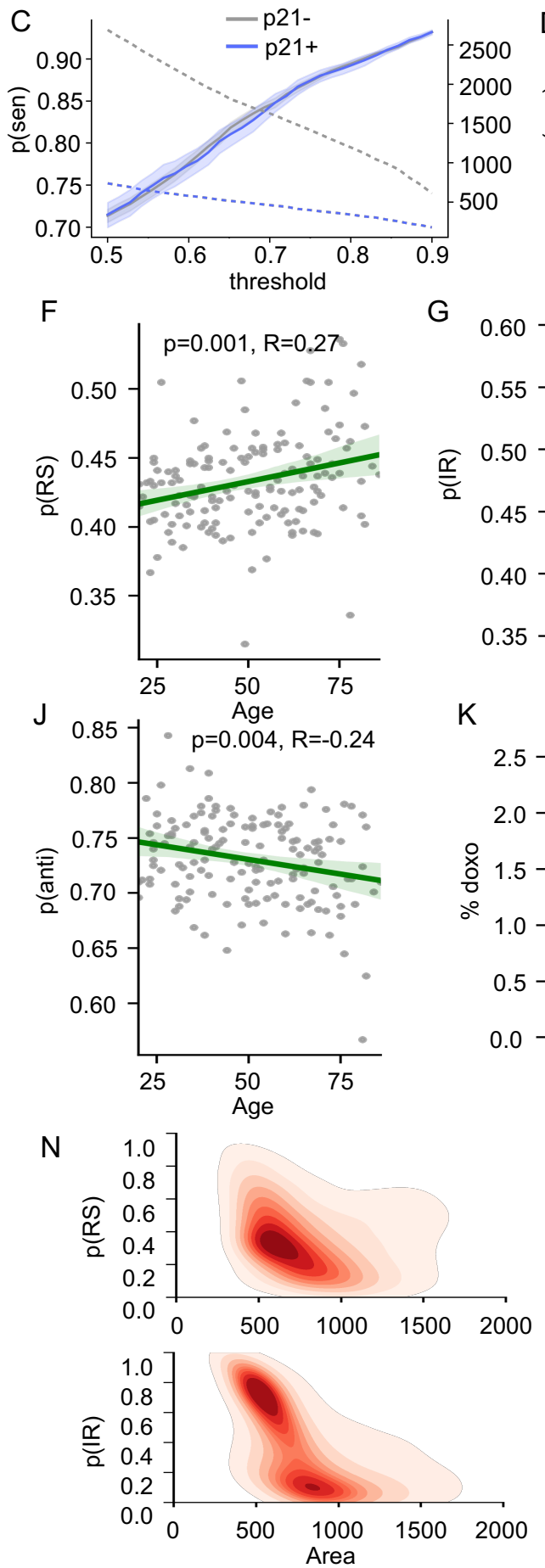

G
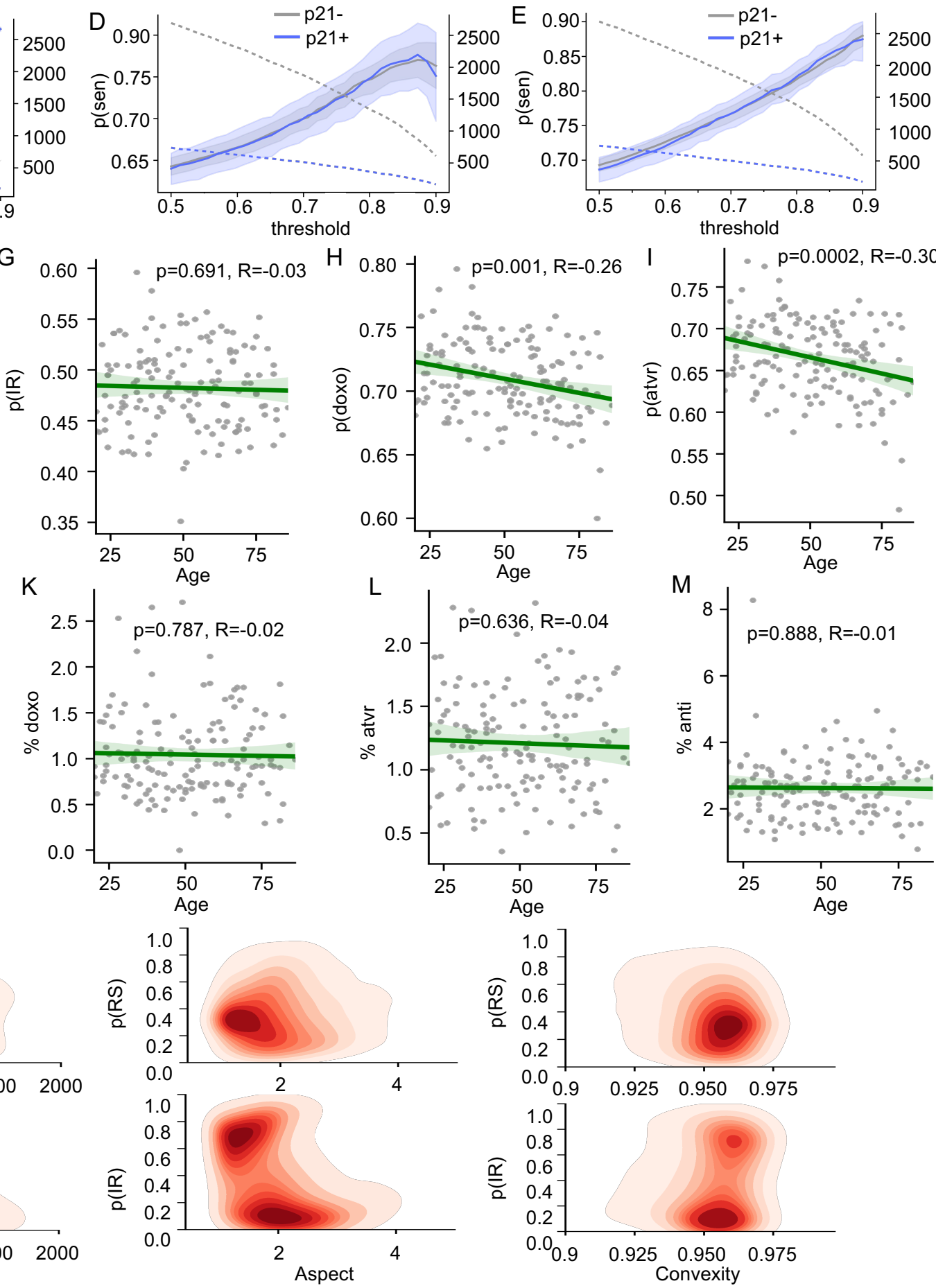

Figure S6 Senescence metrics in human dermal fibroblasts. a Sample of human dermis, stained with H\&E and DAB for p21. b Predicted probability of senescence from IR model and number of nuclei for p21- and p21+ nuclei in human dermis. c Predicted probability of senescence from Doxorubicin model and number of nuclei for $\mathrm{p} 21$ - and p21+ nuclei in human dermis. d Predicted probability of senescence from ATV/r modeland number of nuclei for p21- and p21+ nuclei in human dermis. e Predicted probability of senescence from antimycin-A model and number of nuclei for p21- and p21+ nuclei in human dermis. $\mathbf{f}$ Predicted probability of RS senescence $(n=169)$. g Predicted probability of IR senescence $(n=169)$. $\mathbf{h}$ Predicted probability of doxorubicin senescence $(n=169)$. i Predicted probability of ATV/r senescence ( $n=169)$. j Predicted probability of antimycin-A senescence $(n=169)$. $\mathbf{k}$ Percent of doxorubicin senescence $(n=169)$. I Percent of ATV/r senescence ( $n=169)$. $m$ Percent of antimycin-A senescence $(n=169)$. $n$ 2D histograms (density heatmaps) of nuclei from the histology slides. 


\begin{tabular}{|c|c|c|c|c|c|c|c|c|c|c|c|c|}
\hline & IR & RS & Doxo & ATV/r & Anti & Uni & IR\% & $\mathrm{RS} \%$ & Doxo\% & ATV/r\% & Anti\% & Uni\% \\
\hline Neoplasm & 0.005 & 0.002 & & & & 0.005 & 0.006 & & & & & \\
\hline Malignancy & 0.018 & 0.058 & & & & 0.008 & & & & & & \\
\hline Osteoporosis (DM819) & & & & & & & 0.025 & & & & & \\
\hline Osteoarthritis (DM170) & & & & & & & & & 0.031 & & & \\
\hline Osteoarthritis (DM199) & & & & & & 0.037 & & & & & & \\
\hline Hypertension (DI109) & & & & & & & & & & & 0.029 & \\
\hline Hearing loss (DH911) & & & & 0.014 & 0.014 & 0.034 & & & & & & \\
\hline Hearing loss (DH919) & & & & & 0.032 & & & & & & & \\
\hline $\begin{array}{l}\text { Cerebral Infarction } \\
\text { (DI639) }\end{array}$ & & & & & & & & & 0.013 & 0.027 & & \\
\hline Hyperlipidemia (DE785) & & & & & & 0.037 & & & & & & \\
\hline $\begin{array}{l}\text { Hypercholestereima } \\
\text { (DE780) }\end{array}$ & & & & 0.048 & 0.047 & & & & & & & 0.044 \\
\hline
\end{tabular}

Figure S8 Table of Significant Conditions and Additional Conditions. a Table of selected conditions with significant $p$ values. 